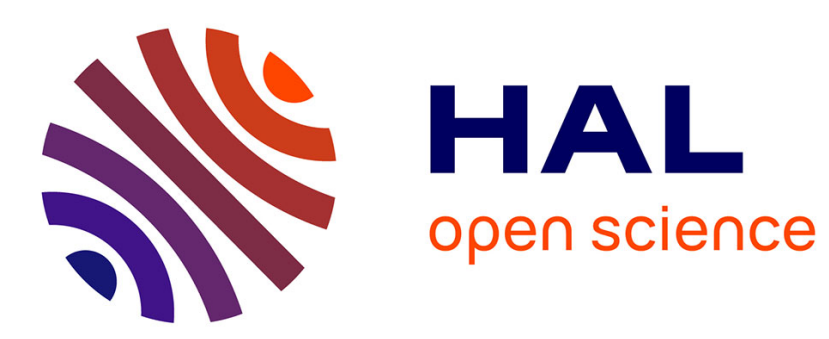

\title{
Azimuthal anisotropy of the Pacific Ocean
}

Alessia Maggi, Eric Debayle, Keith Priestley, Guilhem Barruol

\section{To cite this version:}

Alessia Maggi, Eric Debayle, Keith Priestley, Guilhem Barruol. Azimuthal anisotropy of the Pacific Ocean. Earth and Planetary Science Letters, 2006, 250 (1-2), pp.53-71. 10.1016/j.epsl.2006.07.010 . hal-00105942

\section{HAL Id: hal-00105942 \\ https://hal.science/hal-00105942}

Submitted on 28 Jan 2017

HAL is a multi-disciplinary open access archive for the deposit and dissemination of scientific research documents, whether they are published or not. The documents may come from teaching and research institutions in France or abroad, or from public or private research centers.
L'archive ouverte pluridisciplinaire HAL, est destinée au dépôt et à la diffusion de documents scientifiques de niveau recherche, publiés ou non, émanant des établissements d'enseignement et de recherche français ou étrangers, des laboratoires publics ou privés. 


\title{
Azimuthal anisotropy of the Pacific region
}

\author{
Alessia Maggi ${ }^{\text {a,* }}$, Eric Debayle ${ }^{\text {a }}$, Keith Priestley ${ }^{b}$, Guilhem Barruol ${ }^{\text {c,d }}$ \\ ${ }^{a}$ CNRS and Université Louis Pasteur, 67084 Strasbourg, France \\ b Bullard Laboratories, University of Cambridge, Cambridge, CB3 OEZ, UK

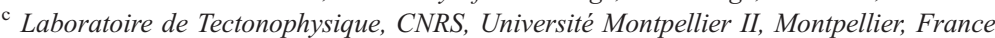 \\ ${ }^{\mathrm{d}}$ Lab. Terre Océan, Université de Polynésie française, Tahiti, French Polynesia
}

Received 23 February 2006; received in revised form 6 July 2006; accepted 10 July 2006

Available online 7 September 2006

\begin{abstract}
Azimuthal anisotropy is the dependence of local seismic properties on the azimuth of propagation. We present the azimuthally anisotropic component of a 3D SV velocity model for the Pacific Ocean, derived from the waveform modeling of over 56,000 multi-mode Rayleigh waves followed by a simultaneous inversion for isotropic and azimuthally anisotropic $v_{\mathrm{sv}}$ structure. The isotropic $v_{\mathrm{sv}}$ model is discussed in a previous paper (A. Maggi, E. Debayle, K. Priestley, G. Barruol, Multi-mode surface waveform tomography of the Pacific Ocean: a close look at the lithospheric cooling signature, Geophys. J. Int. 166 (3) (2006). doi:10.1111/ j.1365-246x.2006.03037.x). The azimuthal anisotropy we find is consistent with the lattice preferred orientation model (LPO): the hypothesis of anisotropy generation in the Earth's mantle by preferential alignment of anisotropic crystals in response to the shear strains induced by mantle flow. At lithospheric depths we find good agreement between fast azimuthal anisotropy orientations and ridge spreading directions recorded by sea-floor magnetic anomalies. At asthenospheric depths we find a strong correlation between fast azimuthal anisotropy orientations and the directions of current plate motions. We observe perturbations in the pattern of seismic anisotropy close to Pacific hot-spots that are consistent with the predictions of numerical models of LPO generation in plume-disturbed plate motion-driven mantle flow. These observations suggest that perturbations in the patterns of azimuthal anisotropy may provide indirect evidence for plume-like upwelling in the mantle.
\end{abstract}

Keywords: Pacific; lithosphere; surface wave; tomography; azimuthal anisotropy; plumes

\section{Introduction}

Seismic anisotropy - the dependence of local seismic propagation characteristics on the direction and polarization of the seismic wavefield - is pervasive throughout the uppermost mantle, and causes effects as diverse as the azimuthal variation of $P_{n}$ velocities in the oceans [2,3], $S$-wave splitting in teleseismic $S K S$ waves [4], the incompatibility of Love and Rayleigh wave dispersion curves with a unique isotropic Earth model $[5,6]$, and the azimuthal variations of surface wave velocities $[7,8]$.

The most likely cause of upper mantle anisotropy is the alignment (lattice preferred orientation, LPO) of intrinsically anisotropic crystals in mantle flow $[9,10]$. Among these crystals, olivine is expected to play an important role due to its abundance in the upper mantle and its strong intrinsic anisotropy. Laboratory experiments and numerical modeling of LPO suggest that simple shear at the base of a moving plate will produce 
anisotropy in olivine with a fast $a$ axis that follows the principal extension direction for modestly deformed olivine aggregates, and aligns with the direction of flow for large deformation $[11,12]$. Although complications are likely to occur under water rich conditions $[13,14]$ or due to the presence of other anisotropic upper mantle minerals such as pyroxene [15], observations of seismic anisotropy are often considered to be useful indicators of mantle flow, and have been much studied in this light $[16,17,12,18-21]$.

In this paper we concentrate on the azimuthal anisotropy of the Pacific Ocean region. Azimuthal anisotropy is the dependence of local seismic propagation characteristics (in our case Rayleigh wave velocities) on the azimuth of propagation. There have been a number of previous studies of azimuthal anisotropy covering this region [22,23,8,24-26,20,21,27]. Nishimura and Forsyth $[23,8]$ were the first to observe and coarsely map Rayleigh wave azimuthal anisotropy in the Pacific, using measurements of fundamental mode phase velocity dispersion. Montagner and Tanimoto [24] obtained global maps of shear wave azimuthal anisotropy by producing maps of fundamental mode Love and Rayleigh wave phase velocity anisotropy, and subsequently inverting them for shear wave elastic parameters with depth. More recently, Montagner [25] has updated the Montagner and Tanimoto [24] study using a much larger data-set $(55,000 v s 4800$ fundamental mode phase velocity dispersion measurements), Trampert and Woodhouse [26] have constructed global maps of phase velocity azimuthal anisotropy from over $\sim 100,000$ phase velocity dispersion measurements, and Beucler and Montagner [28] have constructed similar maps from fewer seismograms $(\sim 19,000)$ but including up to the second surface wave overtone. At the regional scale, Smith et al.[27] have recently investigated in detail the azimuthal variations of Rayleigh wave group velocities in the period range 25-150 s. These studies are mostly based on the analysis of phase or group dispersion curves of fundamental mode surface waves. They conclude that at large spatial scales the directions of fast azimuthal anisotropy are consistent with fossil seafloor spreading in the shallow mantle (down to $\sim 100 \mathrm{~km}$ depth), and that deeper azimuthal anisotropy (down to $\sim 250 \mathrm{~km}$ depth) is strongly related to the current absolute plate motion (APM). Mantle flow models that include the effects of density driven mantle dynamics $[21,20]$ slightly improve the fit to the azimuthal anisotropy observations compared to simple plate-motion models, but they depend strongly on density and viscosity models of the mantle, neither of which are as yet very well determined.
In a previous paper [1], we presented the isotropic component of a 3D SV velocity model for the Pacific Ocean, derived from the waveform modeling of over 56,000 multi-mode Rayleigh waves followed by a simultaneous inversion for isotropic and azimuthally anisotropic $v_{\mathrm{sv}}$ structure. In this paper, we discuss the azimuthally anisotropic results of that tomographic study. Our waveform modeling approach $[29,30]$ extracts more of the information contained in the Rayleigh wave seismograms than the phase or group velocity methods previously used in the Pacific oceans. Indeed, our waveform approach is not restricted to the analysis of the fundamental mode of surface waves, but allows us to include up to the fourth higher surface wave mode in the period range 50-160 s. Surface wave higher modes provide us with additional resolution over the whole depth range of inversion, especially at the bottom of the lithosphere and in the asthenosphere where the fundamental mode starts to lose sensitivity. Furthermore, our tomographic method inverts directly for shear wave isotropic SV velocity and azimuthal anisotropy at each depth and geographic location $[31,32]$. This approach provides better vertical resolution compared to group or phase dispersion anisotropic maps $[27,26,20]$, which represent a weighted average of the structure and anisotropy over a frequency-dependant depth interval. Finally, our data-set provides enhanced coverage of the southern and eastern Pacific Ocean compared to previous studies, thanks to data from ten temporary stations deployed in French Polynesia as part of the Pacific Lithosphere and Upper Mantle Experiment [33].

In this paper, we discuss the distribution of azimuthal anisotropy in the Pacific Ocean lithosphere and asthenosphere. We confirm in particular that the azimuthal anisotropy observed in the Pacific upper mantle agrees to first order with a model of LPO caused by the shearing of mantle materials during the formation and translation of the tectonic plates. We also show that this plate motion induced azimuthal anisotropy is perturbed by upwelling mantle plumes in the vicinity of known Pacific hot-spots. Finally, we compare our results with observations of radial anisotropy of the Pacific upper mantle.

\section{Data, method and sensitivity}

\subsection{Data}

We have analysed vertical component Rayleigh wave seismograms for all earthquakes of magnitude greater than $M_{W} 5.5$ that occurred between 1977 and 2003, and for which the $R 1$ portion of the surface waves propagated exclusively in the Pacific Ocean hemisphere. 
The vast majority of our recordings were obtained from the IRIS (Incorporated Research Institutions for Seismology) and GEOSCOPE databases, with the addition of recordings from a two year deployment of 10 seismograph stations in French Polynesia [33]. These Polynesian records increased the coverage in the South Pacific by $25 \%$, allowing us to improve our resolution of this region compared to previous studies. Our full data-set contains several hundred thousand seismograms.

The automated multi-mode surface waveform inversion which we used for the waveform inversion step of our tomography [30] imposes stringent data quality requirements and rejects paths for which the waveform inversion does not converge. A total of 56,217 waveforms met all of the requirements and were included in our tomographic inversion. We grouped the path-averaged models resulting from these waveforms using a cluster radius of $200 \mathrm{~km}$ around both epicenter and station, and treated the shear wave models within each cluster as independent measurements of the average shear wave profile along their common great-circle path [1]. Our clustering procedure led to a significantly smoother path coverage, to more accurate measurements of the path-averaged shear wave velocity structure, and to improved estimates of the uncertainties associated with these measurements. Fig. 1a shows the density of the resulting 15,165 clustered paths.

\subsection{Method}

We use a two-stage surface wave tomography procedure that has proved to be successful in a number of regional-scale tomographic studies [31,34-37], to invert simultaneously for isotropic SV velocity and azimuthal anisotropy in the Pacific region.

In the first stage, we model vertical component long period multi-mode Rayleigh wave seismograms in the period range $50-160 \mathrm{~s}$ to obtain $1 \mathrm{D}$ average shear wave velocity models, using the method of secondary observables originally proposed by Cara and Lévêque [29], and automated by Debayle [30]. We invert for the upper mantle structure only, and use as our starting model a smoothed version of the 1D $v_{\mathrm{sv}}$ profile from PREM [38], with the crustal portion adapted to each path by averaging the 3SMAC [39] model along the great circle path. The shear slowness of the 1D models obtained from the waveform inversion can be regarded as the average of the local shear slowness structure along the great-circle path between source and receiver [34] :

$\frac{1}{v_{\mathrm{sv}}^{\mathrm{es}}(z)}=\frac{1}{L^{\mathrm{es}}} \int_{e s} \frac{1}{v_{\mathrm{sv}}^{\mathrm{loc}}(z)} \mathrm{d} s$

where $L^{\mathrm{es}}$ is the epicenter-station length, and $v_{\mathrm{sv}}^{\mathrm{es}}(z)$ and $v_{\mathrm{sv}}^{\text {loc }}(z)$ are respectively the shear velocity obtained from the waveform inversion and the local shear velocity at depth $z$.

In the second stage, we invert the shear slowness of the average 1D models tomographically using Montagner's algorithm [40], recently optimized for massive data-sets by Debayle and Sambridge [32]; before inversion we cluster the $1 \mathrm{D}$ models geographically to improve the smoothness of the data coverage and to account for the uncertainties introduced by errors in earthquake focal parameters. Our tomographic procedure exploits the azimuthal dependence of the local phase and group velocity at period $T$ for Love and Rayleigh waves in a slightly anisotropic medium [41]:

$$
\begin{aligned}
C(T)= & C_{0}(T)+C_{1}(T) \cos 2 \theta+C_{2}(T) \sin 2 \theta \\
& +C_{3}(T) \cos 4 \theta+C_{4}(T) \sin 4 \theta,
\end{aligned}
$$

where $C_{0}(T)$ is the isotropic term representing the local value of the phase or group velocity, $\theta$ is the azimuth and

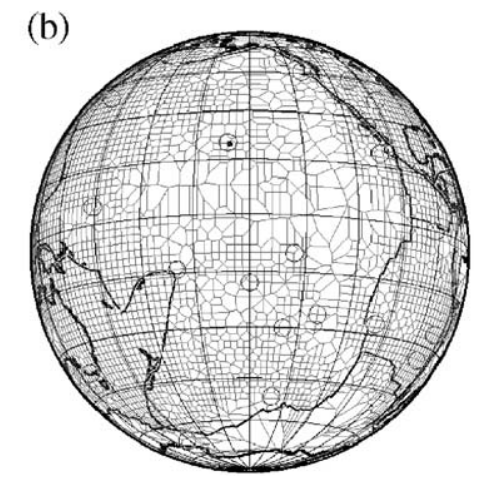

Fig. 1. (a) Density of clustered paths. Color scale indicates number of paths per unit area (the area of a $1^{\circ} \times 1^{\circ}$ cell at the equator). Plate boundaries are indicated by thick black lines; mantle plumes as cataloged by [67] are indicated by circles. (b) Optimized Voronoi diagram for the resolution of Rayleigh wave azimuthal anisotropy.
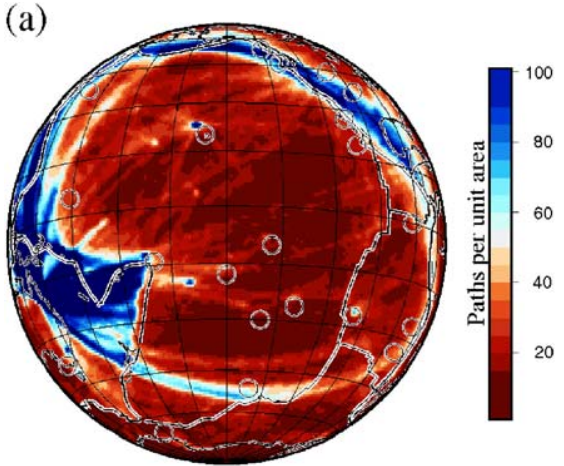
$C_{1}(T), C_{2}(T), C_{3}(T), C_{4}(T)$ are the azimuthal coefficients. From a set of path-average phase or group velocity curves, it is possible to invert for the local $C_{n}(T)$ coefficients and to build tomographic maps for the lateral variations and azimuthal anisotropy in group or phase velocity. Montagner and Nataf [42] have shown that the azimuthal coefficients $C_{1}(T), C_{2}(T), C_{3}(T), C_{4}(T)$ depend on several combinations of the elastic parameters via a set of partial derivatives proportional to the partial derivatives of a transversely isotropic medium with a vertical axis of symmetry; they have also shown that azimuthal anisotropy as a function of depth in the mantle can be retrieved from the observed azimuthal variations of Love and Rayleigh wave velocities. Lévêque et al. [31] described in detail the procedure required to retrieve the distribution of heterogeneities and anisotropy as a function of depth, from the $v_{\text {sv }}$ path-averaged models we obtain in our waveform inversion stage. They also show that, in the long period approximation, the velocity of horizontally propagating SV waves has a $2 \theta$ dependence on the propagation azimuth, controlled by the anisotropic parameters $G_{c}$ and $G_{s}$ [42].We therefore follow [31] and express the local $v_{\mathrm{sv}}$ perturbation at each depth and at each geographical point, $\delta v_{\mathrm{sv}}^{\text {loc }}$, as:

$\delta v_{\mathrm{sv}}^{\mathrm{loc}}=\delta v_{\mathrm{sv}}^{0}+A_{1} \cos 2 \theta+A_{2} \sin 2 \theta$,

where $\delta v_{\mathrm{sv}}^{0}$ is the isotropic perturbation of the SV velocity, $\theta$ is the azimuth, $A_{1}=\left(G_{c} / 2 \rho v_{\mathrm{sv}}^{0}\right), A_{2}=\left(G_{s} / 2 \rho v_{\mathrm{sv}}^{0}\right)$, and $\rho$ is the density. This equation only contains isotropic and $2 \theta$ terms, in contrast with the phase velocity azimuthal dependency Eq. (2), which also contains $4 \theta$ terms. We discuss the effect on the inversion of neglecting the $4 \theta$ contribution to Rayleigh wave anisotropy in Section 2.3.

We invert for the parameters $\delta v_{\mathrm{sv}}^{0}, A_{1}$ and $A_{2}$ using the continuous regionalization algorithm of Debayle and Sambridge [32]. The lateral smoothness of the tomographic model is ensured by correlating neighbouring points using a Gaussian a priori covariance function with the form:

$C_{0}\left(r, r^{\prime}\right)=\sigma(r) \sigma\left(r^{\prime}\right) \exp \left[\frac{-\Delta_{r r^{\prime}}^{2}}{2 L_{\text {corr }}^{2}}\right]$,

where $\Delta_{r r^{\prime}}$ is the distance between 2 geographic points $r$ and $r^{\prime}$. The degree of smoothing is controlled by the horizontal correlation length, $L_{\text {corr }}$, which determines the Gaussian's width; the amplitude of the perturbations in the inverted model is controlled by the a priori standard deviation, $\sigma(r)$, which determines the Gaussian's ampli- tude. This regularization scheme has the advantage of using only parameters that are directly related to the physical characteristics of the output model. We choose a standard deviation of $0.05 \mathrm{~km} \mathrm{~s}^{-1}$ and $0.005 \mathrm{~km} \mathrm{~s}^{-1}$ for the isotropic and anisotropic parameters respectively, indicating that we expect 10 times more isotropic structure than anisotropic structure, a ratio that is commonly used in azimuthal anisotropy studies [26,36]. The choice of an appropriate correlation length depends on the path coverage (Fig. 1) and on the period range of inversion. We chose a value of $L_{\text {corr }}$ such that the effective cross-section of the paths with width $2 L_{\text {corr }}$ ensured a good coverage of the area under study. This criterion was satisfied by a value of $L_{\text {corr }}$ of $400 \mathrm{~km}$, which corresponds to the average wavelength of our data-set. Increasing $L_{\text {corr }}$ by a factor of two leads to smoother images in both the isotropic and anisotropic signals, and larger amplitudes of azimuthal anisotropy but leaves the overall pattern of anomalies unchanged (Fig. 2).

\subsection{Approximations involved in the inversion of} azimuthal anisotropy, and the effect of neglecting the $4 \theta$ contribution

Montagner and Nataf [42] show that, omitting variations in density, the phase velocity of Rayleigh waves propagating in a flat structure at a given azimuth $\theta$ depends only on four combinations of the elastic parameters and four partial derivatives. Using their notation:

$$
\begin{aligned}
\delta C_{\mathrm{R}}= & \frac{\partial C_{\mathrm{R}}}{\partial A}\left(\delta A+B_{c} \cos 2 \theta+B_{s} \sin 2 \theta+C_{c} \cos 4 \theta\right. \\
& \left.+C_{s} \sin 4 \theta\right)+\frac{\partial C_{\mathrm{R}}}{\partial C} \delta C+\frac{\partial C_{\mathrm{R}}}{\partial F}(\delta F \\
& \left.+H_{c} \cos 2 \theta+H_{s} \sin 2 \theta\right)+\frac{\partial C_{\mathrm{R}}}{\partial L}(\delta L \\
& \left.+G_{c} \cos 2 \theta+G_{s} \sin 2 \theta\right),
\end{aligned}
$$

where $C_{\mathrm{R}}$ is the phase velocity of Rayleigh wave $21 \mathrm{~s}$, $A, C, F$ and $L$ are the transverse isotropy elastic parameters, and $C_{c}, C_{s}, B_{c}, B_{s}, H_{c}, H_{s}, G_{c}$ and $G_{s}$ are the elastic parameters describing azimuthal anisotropy. All combinations of elastic parameters in Eq. (5) contain a transverse isotropy term $(A, C, F$ or $L)$, and all but one contain an azimuthal anisotropy term. Among the four partial derivatives in this equation, $\partial C_{\mathrm{R}} / \partial L$ largely dominates the others [42]. Therefore, the combination of elastic parameters that is best resolved by Rayleigh waves is the last term

$\delta \hat{L}=\delta L+G_{c} \cos 2 \theta+G_{S} \sin 2 \theta$. 
(a) $\mathrm{L}=400 \mathrm{~km}$

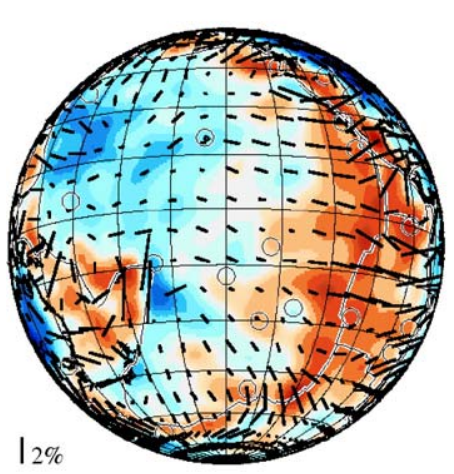

(b) $\mathrm{L}=800 \mathrm{~km}$

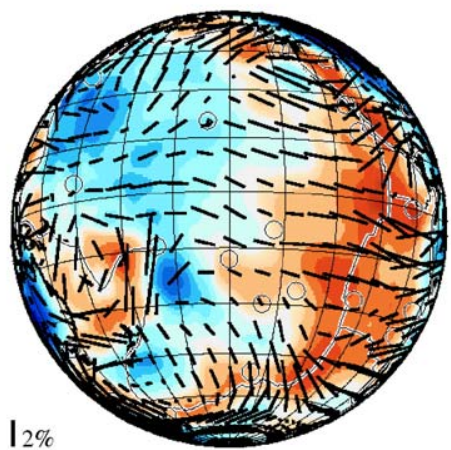

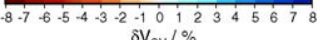

Fig. 2. Inversion results at $100 \mathrm{~km}$ depth using (a) $L_{\mathrm{corr}}=400 \mathrm{~km}$, and (b) $800 \mathrm{~km}$. Throughout this paper, isotropic $v_{\mathrm{sv}}$ values and Rayleigh wave phase velocity values are plotted as percentage variations with respect to a 1D Oceanic Reference Model (ORM), derived by averaging the tomographic model over oceanic regions with age between 30 and $70 \mathrm{Ma}$ and oceanic depth between 4500 and $5000 \mathrm{~m}$ [68,1]; azimuthal anisotropy is denoted by black bars oriented in the local fast propagation direction whose length is proportional to the amplitude in percent of peak to peak anisotropy given by $2 \sqrt{A_{1}^{2}+A_{2}^{2}} / v_{s v}^{0}$, where $v_{\mathrm{sv}}^{0}, A_{1}, A_{2}$ are local values of the isotropic and anisotropic shear wave parameters from Eq. (3).

Lévêque et al. [31] show that, in the long period approximation, $\delta \hat{\mathrm{L}}$ controls the velocity of horizontally propagating SV waves via

$\delta v_{\mathrm{sv}}=\frac{\delta \hat{L}}{2 v_{0} \rho}$

where $v_{0}$ is shear wave velocity in the reference isotropic medium, and $\rho$ is its density. In the waveform inversion stage of our tomographic procedure, we use secondary observables of multi-mode Rayleigh waves to constrain path-averaged values of $\delta \hat{\mathrm{L}}$, and then interpret them as variations in the path-averaged velocity of horizontally propagating SV waves [29]. As $\delta \hat{\mathrm{L}}$ itself only has a $2 \theta$ dependence, we can invert the path-averaged $v_{\mathrm{sv}}$ models using Eq. (3) which has only isotropic and $2 \theta$ terms.

In the waveform inversion stage we have neglected the influence of the partial derivatives with respect to the elastic parameters $A, C$ and $F$. This approximation is common in inversions of Rayleigh waves alone, as they are restricted to the inversion of SV wave velocity only. Inverting for all other terms, with or without a priori coupling between the elastic parameters, does not change the conclusions obtained by inverting only for the best resolved term [8]. In the tomographic inversion stage, neglecting these terms implies ignoring the $4 \theta$ terms in Eq. (2) which are related to the $C_{s}$ and $C_{c}$ terms in Eq. (5) it also implies ignoring the contribution to the $2 \theta$ terms in Eq. (2) of the $B_{c}, B_{s}, H_{c}$ and $H_{s}$ terms from Eq. (5) For the rest of this section we demonstrate that neglecting these terms does not affect our retrieval of $G_{c}$ and $G_{s}$, the parameters that describe the azimuthal anisotropy variations of SV wave velocity in a weakly but fully anisotropic medium.

We first estimate the effect of neglecting the $4 \theta$ terms in Eq. (2) We follow Kennett and Yoshizawa [43] and regard our path-averaged $v_{\mathrm{sv}}$ models as a summary of multi-mode dispersion curves. We predict phase slowness as a function of period from the $1 \mathrm{D} v_{\mathrm{sv}}$ models, then produce two sets of azimuthally anisotropic phase velocity maps using in the first case only the isotropic and $2 \theta$ terms from Eq. (2) and in the second case the isotropic, $2 \theta$ and $4 \theta$ terms. The phase maps are produced using the continuous regionalization algorithm of Debayle and Sambridge [32], the same algorithm we use in our $v_{\mathrm{sv}}$ inversion but this time applied to Eq. (2) We use an $a$ priori correlation length $L_{\mathrm{corr}}=400 \mathrm{~km}$, and $a$ priori model variances $\sigma_{M}=0.05 \mathrm{~km} \mathrm{~s}^{-1}$ for the isotropic term $C_{0}$, and $\sigma_{M}=0.005 \mathrm{~km} \mathrm{~s}^{-1}$ for the anisotropic terms $C_{1}, C_{2}, C_{3}$ and $C_{4}$. These values are the same as those we have adopted in the $v_{\mathrm{sv}}$ inversion for the equivalent isotropic and $2 \theta$ terms of Eq. (3) Note that in the phase velocity inversions we are giving equal weight to the $2 \theta$ and $4 \theta$ terms. The phase velocity maps we produced using all terms in Eq. (2), like those of Trampert and Woodhouse [26], contain a significant $4 \theta$ component, with an amplitude that equals that of the $2 \theta$ component in some regions. However, the $2 \theta$ component remains largely unchanged when compared with the maps produced using only the isotropic and $2 \theta$ terms of Eq. (2) Fig. 3 shows a comparison between the $2 \theta$ 
components of the two phase velocity inversions at 50 and $140 \mathrm{~s}$. The similarity between the two inversions is also observed at other periods, indicating that neglecting the $4 \theta$ terms does not influence the retrieval of the $2 \theta$ azimuthal anisotropy signal. This is equivalent to saying that neglecting the $C_{c}$ and $C_{s}$ terms in Eq. (5) has only a weak influence on the retrieval of the $G_{c}$ and $G_{s}$ terms.

We now estimate the effect of neglecting the $2 \theta$ contributions of $H_{c}, H_{s}, B_{c}$ and $B_{s}$ in Eq. (5). In our phase velocity maps (Fig. 3), these elastic parameters contribute, along with $G_{c}$ and $G_{s}$, to the $2 \theta$ azimuthal anisotropy signal. We can compare these phase velocity maps with the Rayleigh wave phase velocities predicted by integrating our $v_{\mathrm{sv}}$ model along the $G$ sensitivity kernels $\left(\partial C_{\mathrm{R}} / \partial L\right)$. Fig. 4 shows a comparison between the $2 \theta$ components of the phase velocity maps (inverted without the $4 \theta$ terms) and the azimuthal anisotropy predicted from $G_{c}$ and $G_{s}$, both at 50 and 140 s period. Once again, we find a good agreement between the
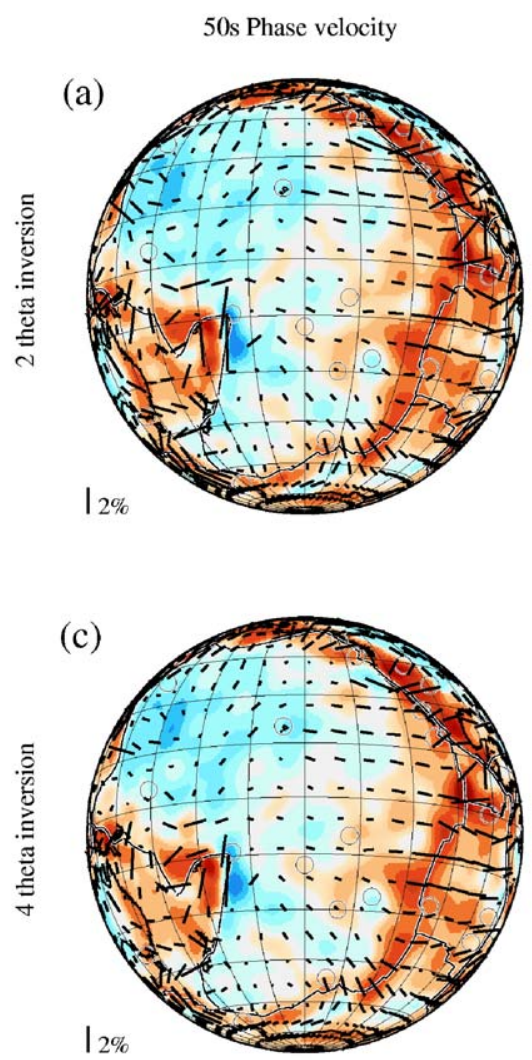

azimuthal anisotropy signals, suggesting that neglecting the $B$ and $H$ contributions does not interfere with the retrieval of the $G_{c}$ and $G_{s}$ terms for the depth and period range of our inversion.

\subsection{Horizontal and vertical resolution}

An elevated path density such as that shown in Fig. 1a does not itself guarantee resolution of azimuthal anisotropy. In order to correctly retrieve both isotropic and azimuthally anisotropic components of $v_{\mathrm{sv}}$ in a given region, we need to resolve the $2 \theta$ Rayleigh wave periodicity of the azimuthally anisotropic signal (see Eq. (3). This requires at least three paths with sufficiently different azimuths crossing each "elementary region" over which the model is required to be smooth. Debayle and Sambridge [32] have shown that it is possible to generate an optimized Voronoi diagram illustrating the shape and size of geographical regions for which this
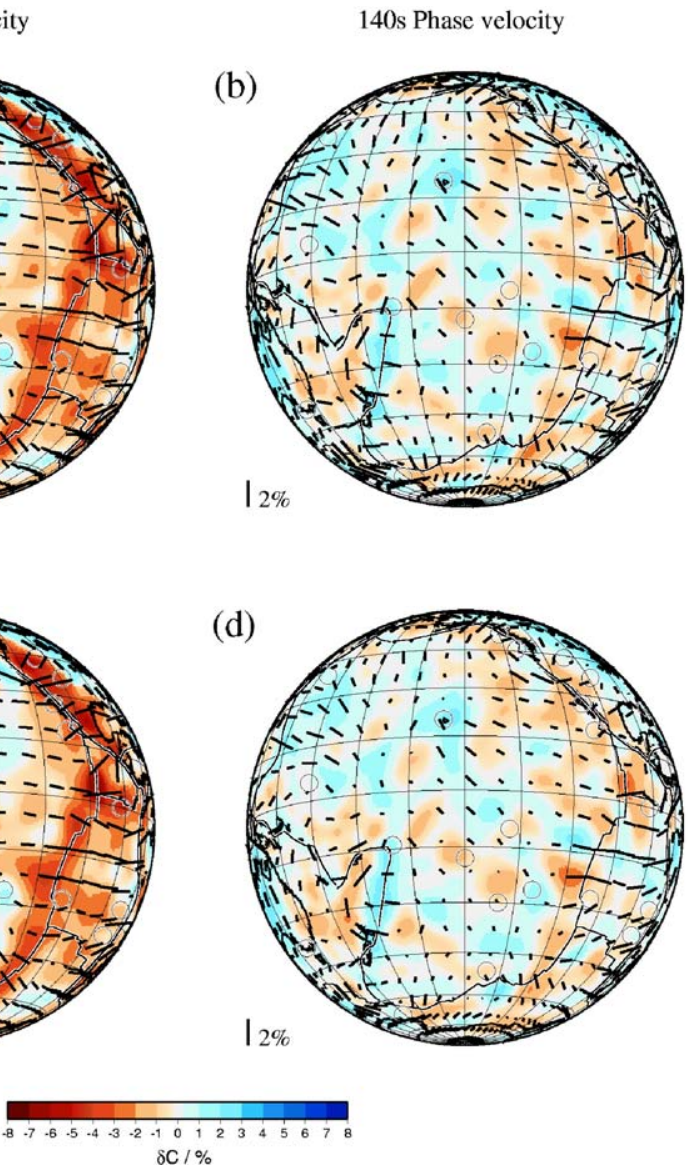

Fig. 3. Effect of neglecting $4 \theta$ terms. All plots show Rayleigh wave phase velocity and the $2 \theta$ component of azimuthal anisotropy, using the same color scale and anisotropic plotting conventions as Fig. 2. (a) $50 \mathrm{~s}$ phase velocity from a $2 \theta$ inversion; (b) $140 \mathrm{~s}$ phase velocity from a $2 \theta$ inversion; (c) $50 \mathrm{~s}$ phase velocity from $2 \theta+4 \theta$ inversion; (c) $140 \mathrm{~s}$ phase velocity from $2 \theta+4 \theta$ inversion. 
geometric criterion is satisfied. Fig. 1b shows the optimized Voronoi diagram for the 15,165 clustered paths used in our tomographic inversion. The size of the Voronoi cells increases from a starting size of $2^{\circ}$ in the western, northern and south-eastern Pacific towards cells of width up to $10-15^{\circ}$ in the central Pacific, indicating that azimuthal coverage, and therefore recovery of azimuthal anisotropy, will be poorest in the central Pacific. Furthermore the shape and elongation of the Voronoi cells gives an indication of the bias in azimuthal anisotropy resolution, as the tomographic procedure resolves spatial variations in anisotropic orientations less well in the direction of the elongation than in the perpendicular direction. The small size and regular shape of Voronoi cells in the normally poorly covered south Pacific region is due to the additional data provided by the PLUME deployment.

For large data-sets, such as that analysed here, the finite width of the surface wave sensitivity zone physically degrades the horizontal resolution from the much higher geometric upper limit determined by the path coverage alone, and illustrated by the path coverage and
Voronoi diagram of Fig. 1. In fact, under the assumptions of ray theory, used throughout this study, surface waves cannot resolve structures smaller than the width of their sensitivity zone. Estimates of this width vary $[44,45]$ and depend on surface wave mode, but generally increase with surface wave period and with increasing path length. In order to optimize our horizontal resolution, we have maximized the proportion of short propagation paths in our data-set, such that $75 \%$ of our paths are shorter than $6000 \mathrm{~km}$. Our chosen correlation length $L_{\text {corr }}=400 \mathrm{~km}$ corresponds to a Gaussian sensitivity zone around each path with a width of $800 \mathrm{~km}$, which is in the center of sensitivity zone width estimates $[44,45]$ for $100 \mathrm{~s}$ fundamental mode Rayleigh waves (the dominant period in our dataset). From the Voronoi diagram of Fig. 1 and the sensitivity zone widths, we estimate that in our best covered regions (e.g. the west Pacific) we obtain a horizontal resolution of $\sim 600 \mathrm{~km}$, but that this worsens to $\sim 1500 \mathrm{~km}$ in the less well covered central Pacific.

Vertical resolution is influenced by the coverage in surface wave modes most sensitive to a given depth. Our
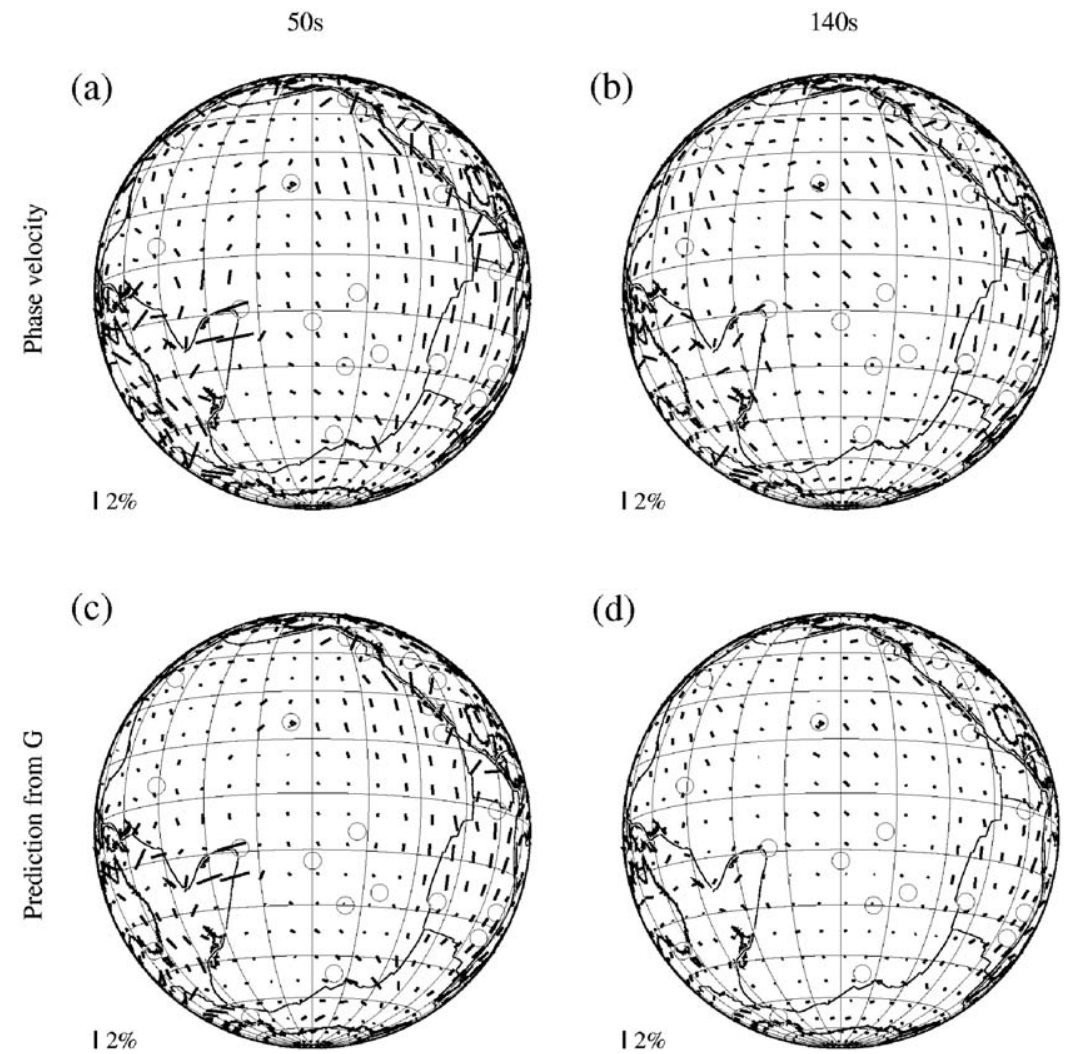

Fig. 4. Effect of neglecting $B$ and $H 2 \theta$ terms. Plots show the $2 \theta$ component of azimuthal anisotropy, using the same anisotropic plotting conventions as Fig. 2. (a) $50 \mathrm{~s}$ phase velocity from a $2 \theta$ inversion; (b) $140 \mathrm{~s}$ phase velocity from a $2 \theta$ inversion; (c) $50 \mathrm{~s}$ phase velocity predicted from the $G_{c}$ and $G_{s}$ of our $v_{\mathrm{sv}}$ inversion; (c) $140 \mathrm{~s}$ phase velocity predicted from the $G_{c}$ and $G_{s}$ of our $v_{\mathrm{sv}}$ inversion. 
higher mode coverage is excellent thanks to the numerous intermediate depth subduction zone earthquakes in our dataset. A full vertical resolution test using our inversion method and a smaller data-set [46] shows that for a $100 \mathrm{~km}$ thick test anomaly, vertical smearing of the isotropic component is small $(\sim 25 \mathrm{~km})$. The method is furthermore able to resolve a $90^{\circ}$ change in azimuthal anisotropy direction over a $50 \mathrm{~km}$ depth interval [36].

\subsection{Synthetic tests}

Imperfect azimuthal coverage leads to incomplete recovery of strong velocity anomalies, and tends to generate trade-off between these strong anomalies and azimuthal anisotropy [31].We analyze the possible trade-off between azimuthal anisotropy and isotropic $v_{\mathrm{sv}}$ by performing synthetic tomographic inversions using our full path coverage. We create synthetic 1D pathaveraged models based on the isotropic 3SMAC model [39] for each of the 15,165 paths of our clustered dataset, and assign to each model the same measurement error as for the real data. We then combine the path average models in a tomographic inversion using the same model a priori information as for the real data. As the input 3SMAC model is isotropic, any azimuthal anisotropy in the output model is an artefact due to the trade-off between anisotropy and shear wave velocity.

The results of this trade-off test are presented in Fig. 5a for a slice at $50 \mathrm{~km}$ depth, and show weak anisotropy $(<0.25 \%)$ being generated by the tomographic inversion, indicative of small amounts of trade-off, except close to strong velocity anomalies (e.g. narrow ridges), where the trade-off anisotropy can reach $1 \%$. In particular, the synthetic test results show that trade-off azimuthal anisotropy directions are consistently normal to the slow $v_{\mathrm{sv}}$ signatures of the mid-ocean ridges. At deeper depths (not shown) where the lateral variations in shear wave velocity are less pronounced, the same test produces smaller amounts of trade-off, and this is concentrated near the fast $v_{\mathrm{sv}}$ signatures of the subduction zones which produce $<1 \%$ trench parallel anisotropy.

It is difficult to compare our trade-off with other tomographic studies of the region, as tests of this kind are seldom published. As an illustration of the typical tradeoffs obtained with this inversion method, Lévêque et al. [31] and Pilidou et al. [35] find ridge-normal trade-off
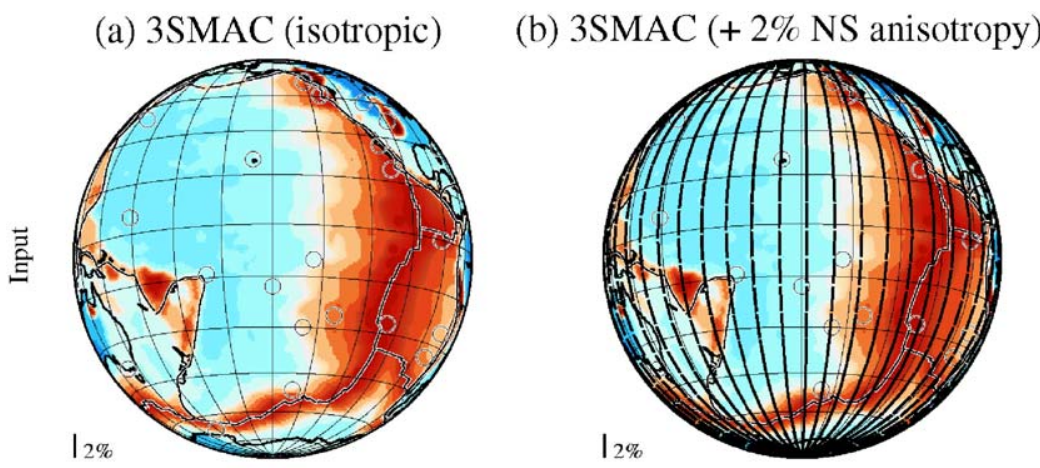

(c) $3 \mathrm{SMAC}(+2 \%$ EW anisotropy)
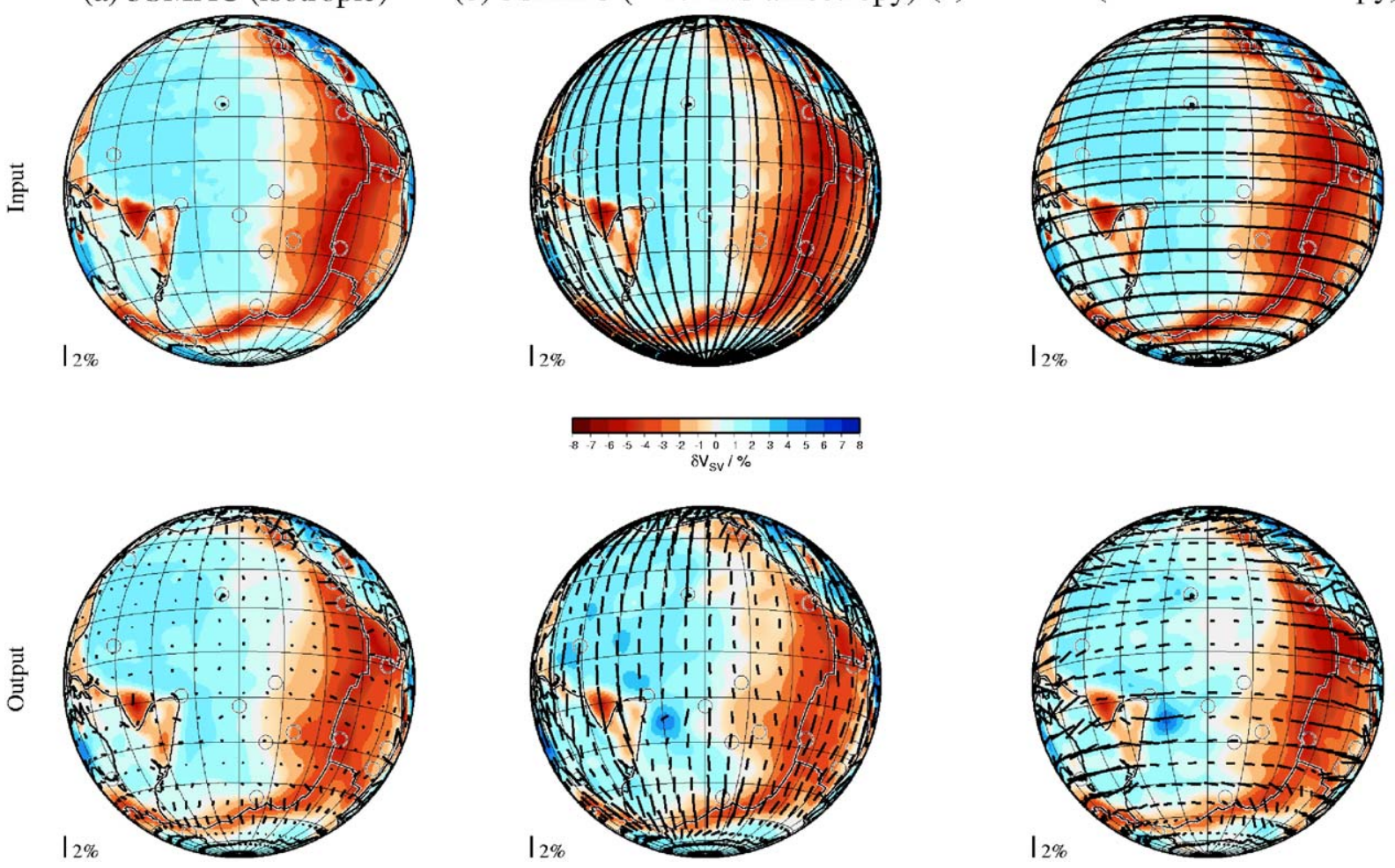

Fig. 5. Trade-off tests between isotropic $v_{\mathrm{sv}}$ and azimuthal anisotropy. Input models (top row) are based on the $50 \mathrm{~km}$ depth isotropic $v_{\mathrm{sv}}$ of $3 \mathrm{SMAC}$ [39], with (a) no azimuthal anisotropy, (b) uniform azimuthal anisotropy with NS fast direction and 2\% amplitude, and (c) uniform azimuthal anisotropy with EW fast direction and 2\% amplitude. Tomographic results for each input model are shown in the bottom row. 
azimuthal anisotropy with amplitudes up to $1 \%$ and $0.7 \%$ in the Indian and Atlantic oceans respectively. Trampert and Woodhouse [26] quantify their trade-off by filtering an isotropic phase velocity model with the resolution kernels calculated for their fully anisotropic inversion, and conclude that the trade-off is negligible overall, without giving a break-down by region or geographical feature. Smith et al. [27] provide no estimate of their trade-off with isotropic structure. Finally Beucler and Montagner [28] perform a combined resolution and trade-off test with non-tectonically realistic input anomalies, and find trade-off anisotropies of up to $0.5 \%$ where their input isotropic anomalies are strongest.

Our ability to recover azimuthal anisotropy is not significantly affected by trade-off with isotropic structure for over $90 \%$ of the study region. However, this trade-off clearly needs to be taken into account when interpreting the azimuthal anisotropy signature of the mid-ocean ridges at shallow depth. Shallow, ridge normal azimuthal anisotropy close to spreading centers is found in most oceanic and global azimuthal anisotropy studies $[31,35,26]$, is consistent with observations of olivine fabric in ophiolites [47], and is considered to be an indicator of ridge-normal mantle flow. As an illustration of the pervasiveness of this ridge-normal signature, Fig. 6 shows a comparison between the $2 \theta$ components of Rayleigh wave phase velocity at $50 \mathrm{~s}$ obtained respectively in this study, and by Trampert and Woodhouse [26]. Both maps show approximately ridgenormal fast directions of azimuthal anisotropy associated with the two major mid-ocean ridges in the region, the East Pacific Rise and the Pacific-Antarctic Ridge,

\section{(a) This study}

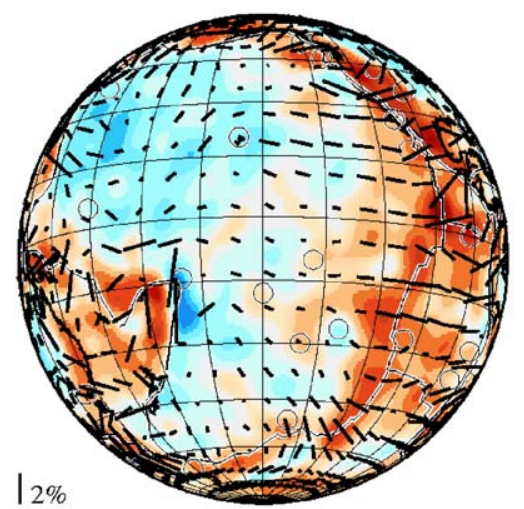

although the lateral smoothing in the Trampert and Woodhouse study is much greater than ours (they expand their anisotropic model in spherical harmonics up to degree 20, with overall lateral resolution of anisotropy up to degree 8). Furthermore, the $v_{\mathrm{sv}}$ images of Fig. 2 and the $50 \mathrm{~s}$ phase velocity images of Fig. 3 illustrate that strong, ridge normal anisotropy is a robust feature of the model, and is unaffected by changes in correlation length or by inclusion of the $4 \theta$ terms in the inversion. The amplitude of ridge normal anisotropy in our phase velocity and $v_{\mathrm{sv}}$ inversions is $\geq 2 \%$ along the East Pacific Rise and the northern half of the Pacific-Antarctic Ridge, or more than twice that generated in the trade-off test.

Although the amplitude we recover from the inversion is greater than that generated in the trade-off test, the fact that the directions are similar leads us to ask to what extent we should interpret this ridge-normal signal; in particular we investigate the possibility that non ridge-normal anisotropy could be rotated into a ridgenormal direction by the tomographic inversion. We therefore test the response of our tomographic inversion to a uniform NS-oriented anisotropic signal (Fig. 5b), and a uniform EW-oriented one (Fig. 5c). Over most of the Pacific, where the tradeoff anomalies are small, the directions of the input anisotropic patterns are well recovered, albeit with a reduction in amplitude of a factor of two in the central and eastern Pacific plate, where the azimuthal coverage is poorest, (large Voronoi cells in Fig. 1b). Along the northern portion of the East Pacific Rise, where the trade-off test of Fig. 5a produces the strongest EW-oriented anisotropy artefacts, the

\section{(b) Trampert \& Woodhouse (2003)}

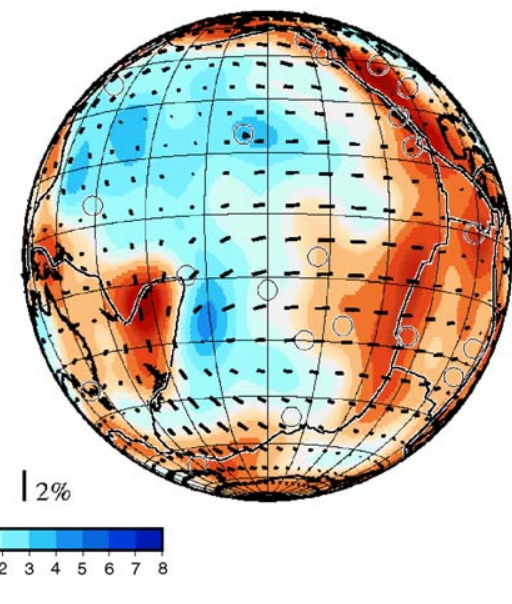

Fig. 6. Comparison between (a) Rayleigh wave phase velocity at $50 \mathrm{~s}$ period from this study ( $2 \theta$ inversion, and (b) Rayleigh wave phase velocity at $40 \mathrm{~s}$ period from [26]. 
tomography cannot recover the NS-oriented input anomalies; however, the trade-off is not strong enough to rotate the output anomalies to an EW direction (Fig. $5 b)$. The portion of East Pacific Rise ridge system between the Galapagos ridge $(\sim 0 \mathrm{~S})$ and the Chile ridge $(\sim 40 \mathrm{~S})$ is not significantly affected by trade-off, suggesting that the ridge-normal signature observed in this region is real. We also observe that the trade-off is weak on the Nazca plate side of the East Pacific Rise, which also shows a strong ridge perpendicular azimuthal anisotropy in the actual model. Further South, along most of the Pacific-Antarctic Ridge, azimuthal coverage is poor, as indicated by large Voronoi cells (Fig. 1b), and the trade-off is strong enough to rotate EW-oriented input anomalies to the NS direction (Fig. 5c).

In conclusion, the presence of ridge-normal azimuthal anisotropy in our tomography within a region demonstrably unaffected by trade-off, the amplitude of this anisotropic signal (twice that obtained in our trade-off test), the observations of ridge normal anisotropy in other surface wave studies [22,24,31,26,27], similar observations made in small scale seismic studies of the EPR $[48,49]$, and discussions of strain and LPO fabric generation at mid-ocean ridges $[50,17,51,52,19]$ all lead us to be confident about our large-scale ridge-normal anisotropy observations, except perhaps in the southern part of our model, along the Pacific-Antarctic Ridge, where the azimuthal coverage is weakest. For regions away from plate boundaries, our azimuthal coverage is largely sufficient for us to discuss the long wavelength variations of the azimuthal anisotropy. We prefer not to interpret the small scale $(<1000 \mathrm{~km})$ details of the azimuthally anisotropic signals, as imperfect azimuthal coverage combined with strong lateral variations in isotropic shear-wave velocity may cause artificial variations in the amplitude, and in some cases direction, of the azimuthal anisotropy over these scales.

\section{Results and discussion}

Fig. 7 shows the results of our tomographic modeling for depths down to $400 \mathrm{~km}$, plotted following the same conventions as Fig. 5. The isotropic $v_{\mathrm{sv}}$ results have been extensively discussed by Maggi et al.[1], so we describe them only briefly here. Our tomographic model shows a high degree of correlation with known tectonic processes: mid-ocean ridges are outlined by slow shear wave velocities down to $100 \mathrm{~km}$ depth; subduction zones are traceable as fast velocities down to $\sim 200 \mathrm{~km}$ depth, with the Japan and Tonga-Kermadec subduction zones visible down to $\sim 250$ and $>400 \mathrm{~km}$ depth respectively; continental cratons display fast velocities down to $150 \mathrm{~km}$, with some roots extending to $200 \mathrm{~km}$. The uppermost Pacific ocean mantle (50-150 km) presents a steady progression of $v_{\mathrm{sv}}$ with ocean-age that is broadly consistent with a half-space mantle cooling model.

In the following discussion, we will be comparing our azimuthal anisotropy model to those obtained in other recent global or regional studies covering the Pacific ocean. Our model describes the azimuthal anisotropy of $v_{\mathrm{sv}}$ as a function of depth, while those we will be comparing it with describe the azimuthal anisotropy of either the phase velocity [26,28] or group velocity [27] of Rayleigh waves as a function of frequency. It is important to bear in mind that phase and group velocity sensitivity kernels are smooth functions of depth between 50 and $400 \mathrm{~km}$ depth, and that they broaden with increasing surface wave period (more severely for phase than for group velocities). The anisotropic phase and group velocity maps published in these studies therefore represent a weighted average of the structure and anisotropy over a frequency dependent depth interval. For reference, $50 \mathrm{~s}, 100 \mathrm{~s}$ and $150 \mathrm{~s}$ phase and group velocity kernels for fundamental mode Rayleigh waves peak at $\sim 60 \mathrm{~km}, \sim 130 \mathrm{~km}$ and $\sim 200 \mathrm{~km}$, and $\sim 50 \mathrm{~km}$, $\sim 100 \mathrm{~km}$ and $\sim 150 \mathrm{~km}$ respectively.

\subsection{Azimuthal anisotropy and plate motions}

The anisotropic structure at shallow depths $(50 \mathrm{~km}$, Fig. 7a) shows strong variations in the orientations of fast $v_{\mathrm{sv}}$ directions across the Pacific plate, from $\mathrm{E}-\mathrm{W}$ in the north-eastern Pacific, to a combination of N-S and NE-SW in the north-western Pacific, to SE-NW in the southernmost Pacific. The East Pacific Rise (EPR) and the Pacific-Antarctic Ridge (PAR) up to the Louisville Hotspot display $\sim 2-3 \%$ ridge-normal anisotropy. Excluding the plate boundaries, the amplitude of shallow azimuthal anisotropy varies between 1-2\% over most of the Pacific plate, except for two regions of low anisotropy in the central Pacific and near the MacDonald hot-spot in the southern Pacific. The Nazca plate displays mostly $\mathrm{E}-\mathrm{W}$ trending anisotropy with an amplitude of $\sim 2 \%$. The apparent factor of two difference in average amplitude of azimuthal anisotropy between the Nazca plate and the central/eastern Pacific plate is similar to the amplitude difference observed in the $\mathrm{E}-\mathrm{W}$ trending synthetic test of Fig. $5 \mathrm{c}$, suggesting that may be due to differences in path density and azimuthal coverage in the two regions (see Fig. 1).

Our pattern of anisotropy can be compared to that obtained by Smith et al. [27] from group velocity measurements and that obtained by Beucler and Montagner 
(a) $50 \mathrm{~km}$

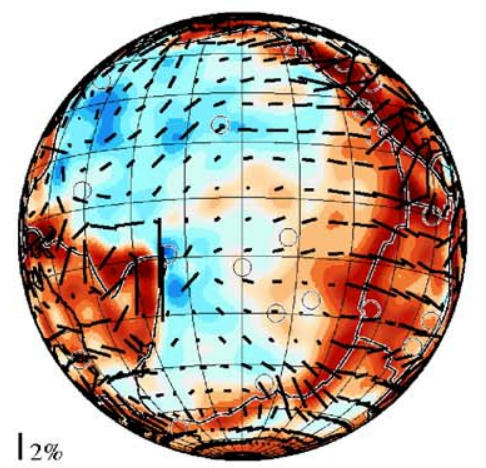

(b) $100 \mathrm{~km}$

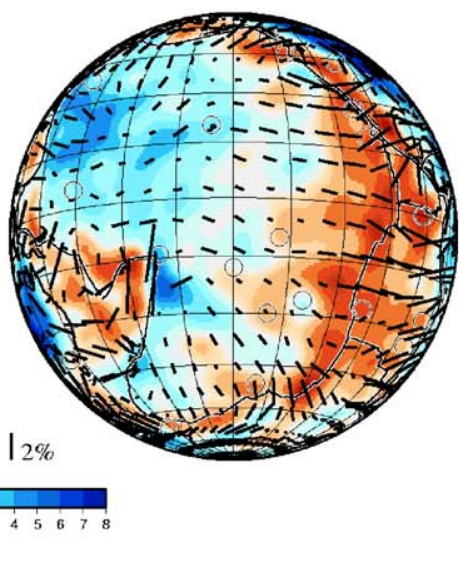

(d) $200 \mathrm{~km}$ (c) $150 \mathrm{~km}$

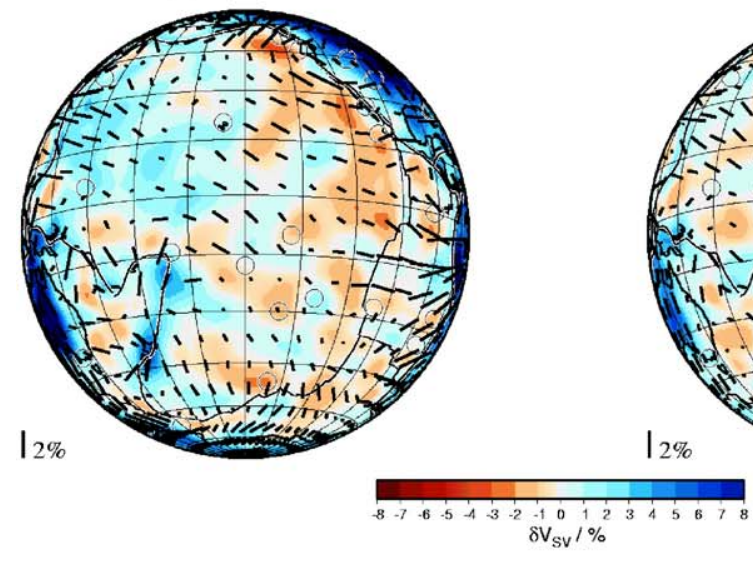

(e) $300 \mathrm{~km}$

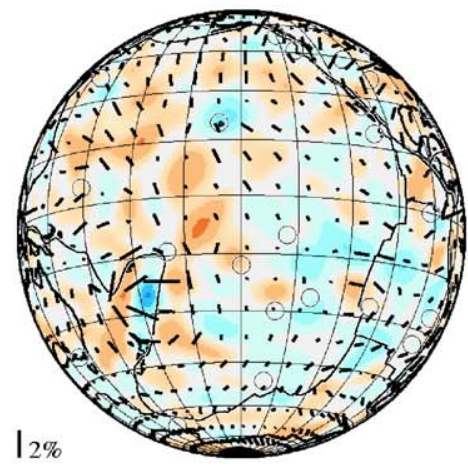

(f) $400 \mathrm{~km}$

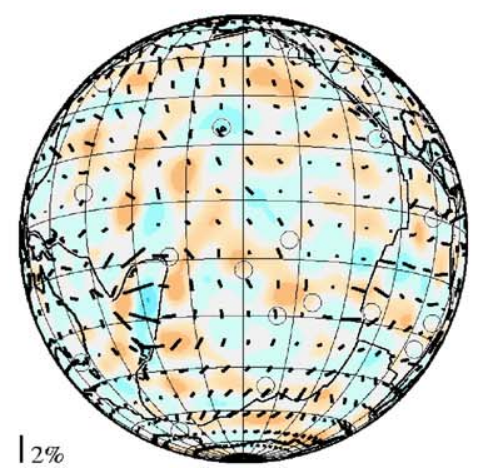

Fig. 7. The tomographic inversion at (a) $50 \mathrm{~km}$, (b) $100 \mathrm{~km}$, (c) $150 \mathrm{~km}$, (d) $200 \mathrm{~km}$, (e) $300 \mathrm{~km}$ and (f) $400 \mathrm{~km}$ depth. For each depth, isotropic $v_{\mathrm{sv}}$ and azimuthal anisotropy are plotted as in Fig. 5.

[28] from phase velocity measurements: their horizontal correlation lengths range from 600 to $1000 \mathrm{~km}$ and, as we have shown in Fig. 2, our overall pattern of azimuthal anisotropy is robust within this range of correlation lengths. Amplitudes of azimuthal anisotropy cannot in general be compared between models as they depend too strongly on the regularization used in the inversions; we will therefore limit ourselves to comparing at most relative amplitudes. Our $50 \mathrm{~km}$ anisotropic structure correlates well in direction and in relative 
amplitude with that of Smith et al. [27] at $50 \mathrm{~s}$ period, once we take into account the variations of amplitude recovery across the region. However, it correlates less well with the $60 \mathrm{~s}$ phase velocity anisotropy of Beucler and Montagner, as at this period they are sensitive to structure ranging from 50 to $100 \mathrm{~km}$ depth (indeed their $60 \mathrm{~s}$ map contains elements from both our 50 and $100 \mathrm{~km}$ depth maps). The comparison with the azimuthal anisotropy of Trampert and Woodhouse [26] is less instructive, as their lateral smoothing is greater than that of the other studies (they resolve only degree 8 anisotropic structure), however it shows that in the long wavelength limit, the anisotropic patterns are consistent (see Fig. 6 for a comparison of the two models in phase velocity space).

According to the commonly held perception of the evolution of the oceanic mantle, the ridge-normal mantle flow signature close to the mid-ocean ridges is expected to 'freeze' into the fabric of the lithosphere, as the latter cools, becomes more viscous, thickens, and moves away from the ridge axis [16]. The fast directions of anisotropy at lithospheric depths are therefore expected to remain perpendicular to the magnetic lineations of the same age (e.g. [8], [27]). Fig. 8 shows a comparison of our azimuthal anisotropy results at $50 \mathrm{~km}$ depth (in the lithosphere of all but the youngest oceanic regions), with catalogued magnetic anomalies [53]. The overall agreement is good in the younger oceans, where fast azimuthal anisotropy directions are consistently perpendicular to magnetic lineations, and most notably so in the northeastern Pacific Ocean region where magnetic lineations are particularly well observed. In the older oceans (120-180 Ma), the magnetic data is more sparse, there is a greater lateral variability in the orientation of magnetic anomalies and a greater discrepancy between magnetic lineations and azimuthal anisotropy directions. Rapid lateral changes in Rayleigh wave azimuthal anisotropy are unresolvable by our inversion because of the width of the sensitivity zone $(500-1000 \mathrm{~km})$ of the 50-160 s period surface waves used in this study. Shorter period Rayleigh waves would be needed to resolve these rapid changes in direction, indeed Smith et al. [27] find a good correlation with magnetic anomalies in the older oceans using $25 \mathrm{~s}$ Rayleigh waves.

At $100 \mathrm{~km}$ depth (Fig. 7b), the directions of anisotropy in the eastern Pacific plate rotate gradually from trending $\mathrm{EW}$ in the northern portion of the plate to trending SE-NW in the southern portion, and continue to rotate until they trend NS in the south-western Pacific plate. The anisotropy of the western Pacific plate at this depth is similar to that found at $50 \mathrm{~km}$ depth. The overall pattern is similar to that found for $100 \mathrm{~s}$ group velocities by Smith et al. [27] As oceanic lithosphere gradually thickens and deepens with age [1], the transition between lithospheric and asthenospheric mantle occurs at progressively deeper depths. We observe in Fig. 7 that the directions of anisotropy, which are heterogeneous in the older oceans at shallow depth, tend to align themselves in longer-wavelength patterns consistent with the directions of plate motion as the depth increases and we pass from the lithosphere into the asthenosphere. It is important to note that trade-off between azimuthal anisotropy and poorly recovered isotropic $v_{\mathrm{sv}}$ anomalies is weaker at these depths than it was at $50 \mathrm{~km}$, as the shear wave anomalies themselves are weaker in amplitude. Weak trade-off is also indicated by lack of correlation between the pattern of isotropic $v_{\mathrm{sv}}$ structure and either the amplitude or direction of azimuthal anisotropy anomalies. Our tomographic maps at 150 and $200 \mathrm{~km}$ depth show similar azimuthal anisotropy patterns, with directions trending NW-SE in the Pacific Plate, E-W in the Nazca Plate and $\mathrm{N}-\mathrm{S}$ in the Australian plate, the approximate directions of absolute plate motion for these plates. These directions are consistent with the $150 \mathrm{~s}$ group velocity results of Smith et al. [27], and the $100 \mathrm{~s}$ phase velocity results of Beucler et al., but not with those of Trampert and Woodhouse [26] who find EW trending fast directions over most of the Pacific plate in their $100 \mathrm{~s}$ phase velocity anisotropy.

Our observations are consistent with the hypothesis of upper mantle anisotropy being caused principally by the preferential orientation of olivine crystals in mantle flow, the lattice preferred orientation (LPO) $[9,10]$. According to numerical modeling of LPO in shear flows [12] and to the LPO measured on mantle rocks [54] (particularly on rocks sampled in ophiolite environments where both lithospheric and asthenospheric fabrics have been brought to the Earth's surface $[55,56]$ ),

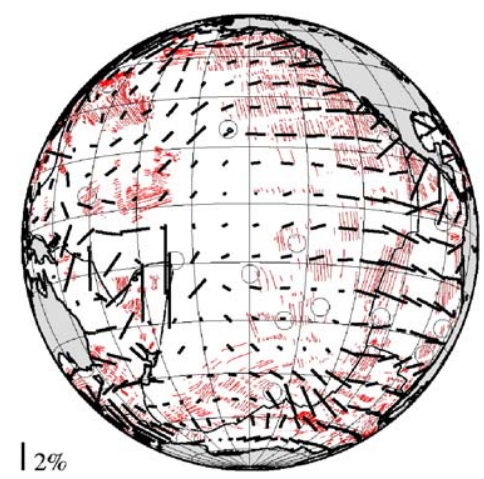

Fig. 8. Shallow anisotropy and oceanic magnetic anomalies. The azimuthal anisotropy results for $50 \mathrm{~km}$ depth plotted above the magnetic anomaly traces. Magnetic anomalies are from [53]. 
the fast [100] or ' $a$ ' crystallographic axes of olivine are maximally concentrated close to the flow direction and the [010] or ' $b$ ' axes are generally concentrated normal to the flow plane. In the case of simple shear flow caused by the drag of a rigid plate over a viscous mantle, the flow direction is the direction of plate motion. The degree to which the fast axes align horizontally in this plane is dependent on the velocity gradient of the flow. Numerical modeling [12] suggests that plate motion induced shear strain accumulates progressively with time and also migrates downward due to the progressive cooling of the mantle and the consequent thickening of the lithosphere. The maximum strain in such a flow is located near the base of the moving plate and falls off with increasing depth. For a 100 Ma old plate, the maximum shear is around $150 \mathrm{~km}$ depth, and shear strain is expected to be attenuated below $200 \mathrm{~km}$ depth. This attenuation is consistent with the observed decrease in amplitude of azimuthal anisotropy in our 300 and $400 \mathrm{~km}$ depth maps (Fig. 7). Our $v_{\text {sv }}$ model, which is constrained at these depths by higher modes, allows us to observe this decrease directly.

In Fig. 9 we map the correlation between azimuthal anisotropy and the directions of absolute plate motion (APM), calculated using the following expression [31]:

$$
|\overline{\text { FastSV }}||\overline{\mathrm{APM}}| \cos \left[2\left(\theta_{\text {FastSV }}-\theta_{\mathrm{APM}}\right)\right],
$$

where $\theta$ indicates the local azimuth of the fast SV and APM directions, and we take as APM directions the NUVEL1 plate motion directions expressed in a no-net rotation frame. At $50 \mathrm{~km}$ depth we see correlation between azimuthal anisotropy and APM at young ages and anti-correlation at older ages. The transition between predominantly correlated and predominantly anti-correlated regions occurs near the $80 \mathrm{Ma}$ isochron, which marks the approximate age at which major plate reorganisation occurred in the Pacific Ocean [57,58]. At ages younger than $80 \mathrm{Ma}$ the fossil ridge spreading directions preserved in the fabric of the lithosphere are in general close to current APM directions, while they differ strongly for lithosphere whose fabric was frozen in before the plate re-organization. An exception is the northeastern Pacific Ocean region, where current APM and fossil ridge spreading directions differ by more than $45^{\circ}$; in this region, the azimuthal anisotropy at $50 \mathrm{~km}$ depth follows as expected the fossil ridge spreading directions (Fig. 8), and is therefore anti-correlated with the APM. As the depth increases, and consequently the proportion of asthenospheric to lithospheric mantle increases outwards from the ridges, the region of good correlation between azimuthal anisotropy and APM also expands outwards from the ridges, until at 150 and $200 \mathrm{~km}$ depth it covers most of the Pacific Ocean. These correlation plots and the correspondence at shallow depths between azimuthal anisotropy directions and magnetic anomalies confirm the hypothesis - dating back to the earliest anisotropic studies [8] and still in use today [27] - of stratification of the anisotropic structure in the Pacific ocean, with fossil anisotropy related to spreading directions in the lithosphere, and anisotropy conforming with current plate motion in the asthenosphere.

\subsection{Azimuthal anisotropy, hot-spots and subduction zones}

In this section we go a step further than previous studies, and discuss those sub-lithospheric regions (depth $\geq 150 \mathrm{~km}$ ) in which the correlation between azimuthal anisotropy and the direction of absolute plate motion breaks down (see 150 and $200 \mathrm{~km}$ depth maps in Fig. 9). Although these anomalous regions are relatively large $(1000-3000 \mathrm{~km})$, because of their depth they are not resolvable by traditional long phase or group dispersion studies, as the long period fundamental mode waves (100-150 s) that sample these depths have sensitivity kernels that average over a large depth range, from 75 to $250 \mathrm{~km}$ for group velocities, and to below $300 \mathrm{~km}$ for phase velocities. These anomalous regions are located in the vicinity of known hot-spots: Bowie, Juan de Fuca, Hawaii, Solomon, Samoa, Galapagos, Easter Island, Society, Marquesas, Mac-Donald and Louisville. The isotropic signature of these hot-spots (Fig. 7) is too weak to create azimuthal anisotropy anomalies by trade-off, nor do these anomalous regions correspond to the regions of weak recovery of azimuthal anisotropy shown in Fig. $5 \mathrm{c}$,d. The depth of these anticorrelated regions, which puts them well below the lithosphere, and their rounded and localized geometry suggests they are not related to the transition between fossil plate motion direction and APM discussed in the previous section. Furthermore, the correspondence between the anomalous regions and the hot-spot locations suggests that the observed perturbation to the platemotion oriented anisotropy may be related to mantle upwelling associated with these hot-spots.

The presence of an upwelling plume is expected to locally perturb the thermal equilibrium of the mantle leading, under certain conditions of mantle flux, plume geometry and mantle viscosity, to erosion of the base of the lithosphere [59] and to reactivation of frozen olivine LPO. Fluid dynamical models of the interaction between an axisymmetric upwelling plume and the simple shear flow induced by a moving plate produce a flow with a roughly parabolic pattern centred over the plume, 
(a) $50 \mathrm{~km}$

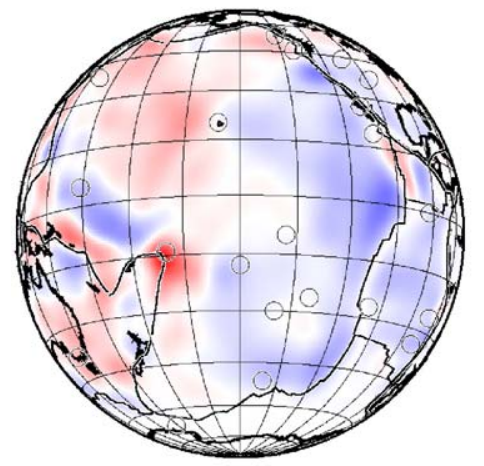

(b) $100 \mathrm{~km}$

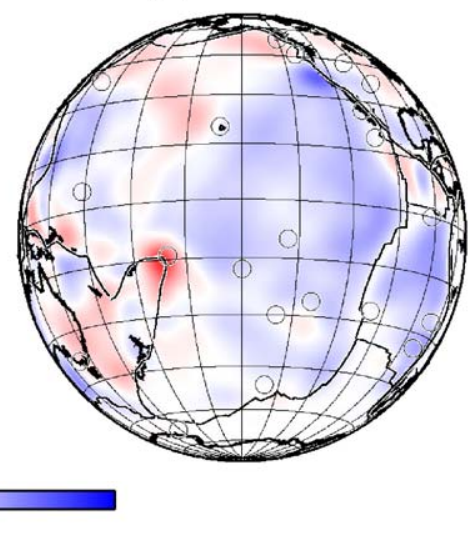

(d) $200 \mathrm{~km}$

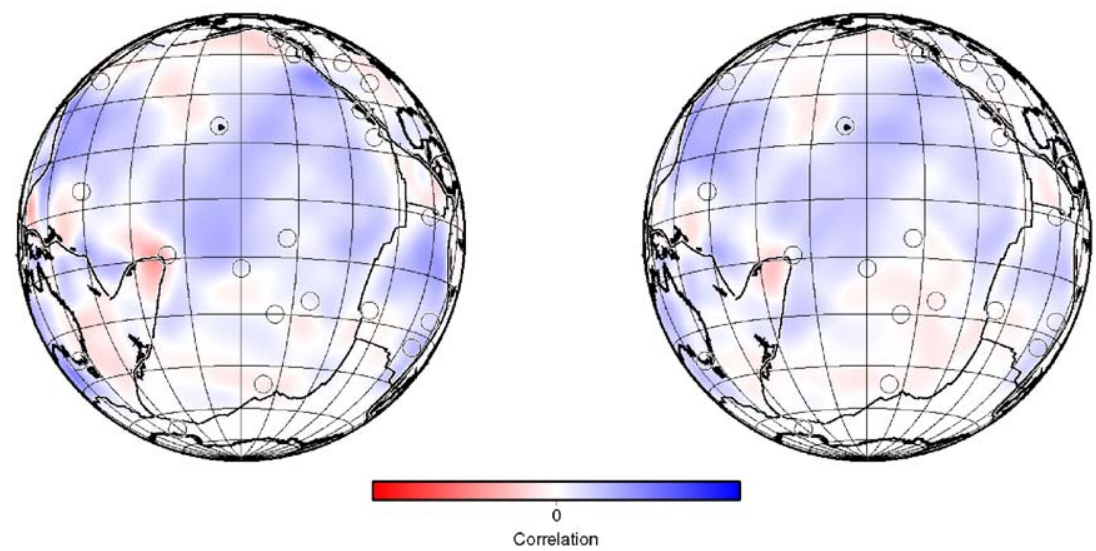

Fig. 9. The correlation between the fast $v_{\mathrm{sv}}$ direction and the direction of absolute plate motion (APM) calculated from NUVEL1 in the no-net rotation reference frame. Areas of strong correlation (anisotropy and APM directions are parallel) are shown in blue; areas of strong anti-correlation (anisotropy and APM directions are perpendicular) are shown in red; areas of weak correlation (either the directions of anisotropy and APM are at $45^{\circ}$ to each other, or one of the two quantities is small) are shown in lighter shades of the two colours.

with a width several times the plume's diameter [60]. Subsequent numerical modeling of LPO in this complex flow pattern using plastic deformation and dynamic recrystallisation models predicts that the fast $a$ axes will not orient themselves along the parabolic flow pattern, and may in certain cases orient themselves almost perpendicular to it. The anisotropic directions may further be perturbed by the presence of water in the upwelling region [13]. The absence of detectable shear wave splitting at seismic stations located on top of known hotspots [61] seems to confirm a perturbation of the pervasive mantle flow at these locations. Although the upwelling plumes themselves are too narrow for us to resolve their intrinsic anisotropic signature, we suggest that the large-scale perturbations of mantle flow induced by the plumes should be detectable using surface wave azimuthal anisotropy.
Fig. 10 shows a synthetic test designed to determine the behaviour of our inversion in the presence of a strong, plume-generated disturbance of the azimuthal anisotropy pattern with the geometry predicted by Kaminski and Ribe ([60], Fig. 7). The base of the input model for the test is the isotropic 3SMAC model [39], on which we have superimposed a uniform $2 \%$ NW-trending azimuthal anisotropy. We have added five plumes to the input model, corresponding to the Hawaii, Society, MacDonald, Marquesas and Pitcairn hot-spots; each plume has an isotropic $v_{\mathrm{sv}}$ anomaly with a radius of $200 \mathrm{~km}$ and an amplitude of $-5 \%$. We have disturbed the uniform anisotropy pattern by imposing a $90^{\circ}$ rotation to the directions of anisotropy in a parabolic region originating at each of the plume locations; the relative dimensions of the plume radius and the parabolic regions are based upon the results of the numerical 
modeling of plume-plate motion interaction by [60]. A slice through the input model at $100 \mathrm{~km}$ depth is shown in Fig. 10a, a plot of the correlation of the input model with 'APM' (i.e. with plate motion in the same direction as the uniform anisotropy and at a uniform rate of $100 \mathrm{~mm} / \mathrm{yr}$, consistent with Pacific plate motion) is shown in Fig. 10b, while the results of the synthetic test are shown in Fig. 10b,d.

After the inversion, the isotropic $v_{\mathrm{sv}}$ signatures of the two visible continental cratons (Australia and North America) have been well recovered, the signatures of the synthetic plumes and of the subduction zones have spread laterally and been attenuated in amplitude, and shear wave velocity variations of up to $\pm 1 \%$ appear in the regions where path coverage is poorest. The main NW-trending azimuthal anisotropy is well-recovered over most of the model, albeit with a reduction in amplitude of up to a factor of two in some regions, similar to that observed in the tests of Fig. $5 \mathrm{c}, \mathrm{d}$. The $90^{\circ}$ perturbation to the anisotropic directions present in the input model is not recovered in the synthetic inversion, however the resulting anisotropic pattern is still perturbed compared to the background NW trending pattern, as is confirmed by the correlation plot (Fig. 10d). Furthermore, the size and amplitude of the anti-correlation anomalies recovered in this test are similar to those found in our tomographic inversion. In a similar test, we were unable to resolve a more subtle radial pattern of anisotropy in the parabolic regions associated with the plumes. These tests indicate that although we do not have sufficient resolution to recover the pattern of a small or medium-scale disturbance in the anisotropy, we are able to detect its presence if the disturbance is severe enough.

Subduction zones are regions in which azimuthal anisotropy is particularly complex. Many regions exhibit fast shear wave splitting direction populations with
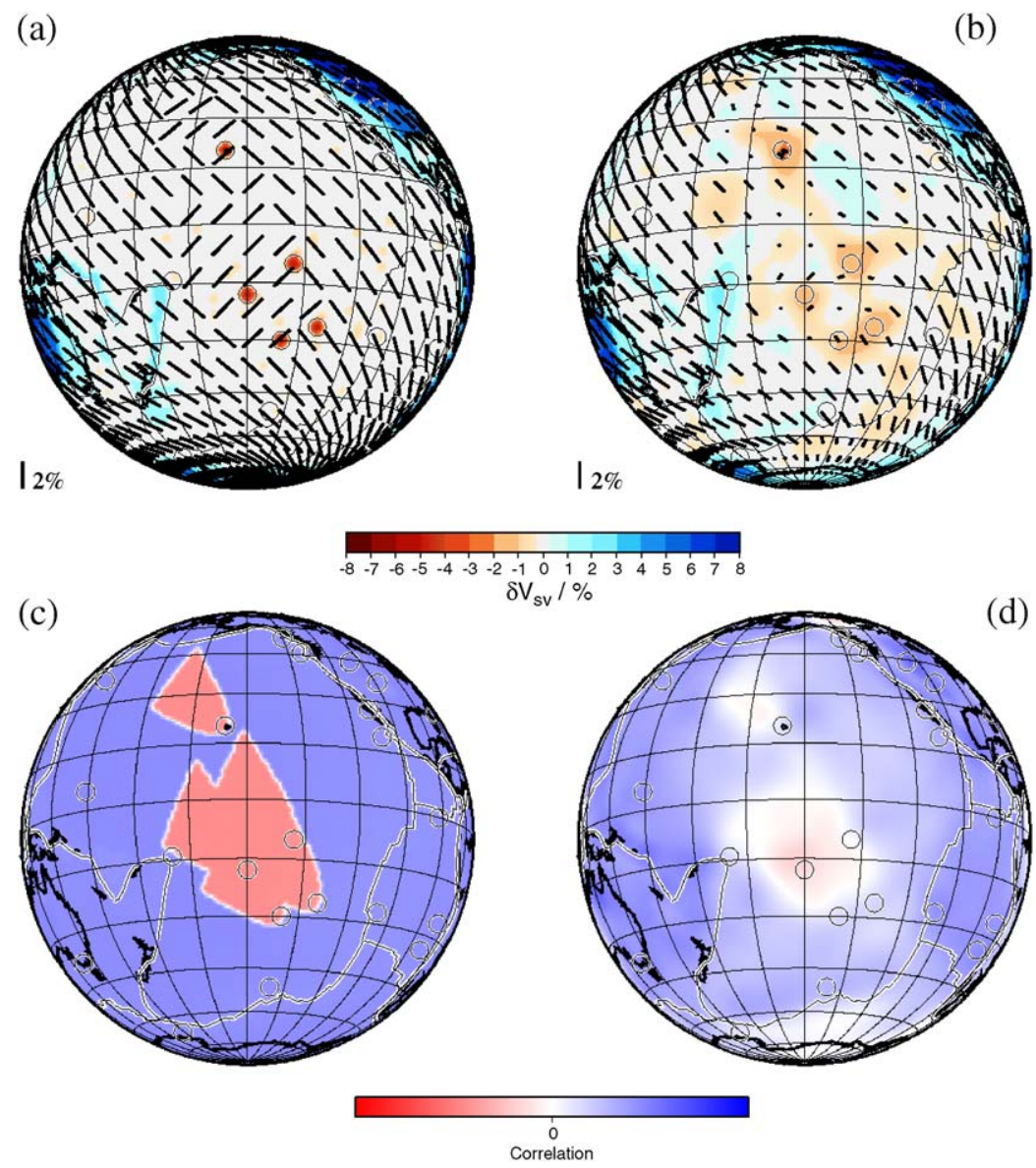

Fig. 10. Synthetic tests for the recovery of plume-related disturbances in azimuthal anisotropy: (a) synthetic input model with uniform $2 \%$ NWtrending anisotropy, 5 plumes of radius $200 \mathrm{~km}$ and 5\% shear wave anomaly, and parabolic regions of rotated anisotropy; (b) output of the synthetic test; (c) input model plotted as correlation with respect to a 'plate motion' (velocity $100 \mathrm{~mm} / \mathrm{yr}$ ) parallel to the background anisotropy; (d) correlation of the output model with the same 'plate motion'. 
orientation both parallel and orthogonal to local trench strike (e.g. Tonga-Fiji, [62]). Interpretation of azimuthal anisotropy near subduction zones is a non-trivial problem, as the mantle flow depends strongly on the interaction between the motions of the subducting plates, and on the rate of retrograde motion [18]. Interpretation is further complicated by experimental studies [13] suggesting that olivine slip systems change under the higher stresses and hydration states likely to be present in subduction zones. Models of LPO orientation in anhydrous and hydrous mantle wedges [63] suggest that the same trench normal mantle flow will create respectively trench normal and trench parallel anisotropy. For the Tonga-Fiji subduction zone, our tomographic model (Fig 7) shows large amplitude trench parallel anisotropy (>4\%) at depths of 50 and $100 \mathrm{~km}$, smaller amplitude (1-2\%) trench parallel anisotropy at $150 \mathrm{~km}$ depth, rotating to trench normal anisotropy with amplitudes ranging from $1 \%$ to $3 \%$ down to at least $400 \mathrm{~km}$ depth. These observations are consistent with a simple mantle wedge, hydrated down to $\sim 150 \mathrm{~km}$ depth (e.g. [63]). Furthermore, although we must recall that trade-off with the fast isotropic shear wave anomalies of the subducting slabs may account for $\sim 1 \%$ of the trenchparallel anisotropic observation, our subduction zone anisotropy observations are broadly consistent with shear wave splitting measurements, which show predominantly trench parallel fast directions near the Tonga-Fiji trench and trench normal fast directions in the back-arc basin [62]. Most studies of seismic anisotropy generation in subduction zone regions are constrained by shear wave splitting measurements $[18,63]$. Our observations suggest that azimuthal

(a) Azimuthal anisotropy

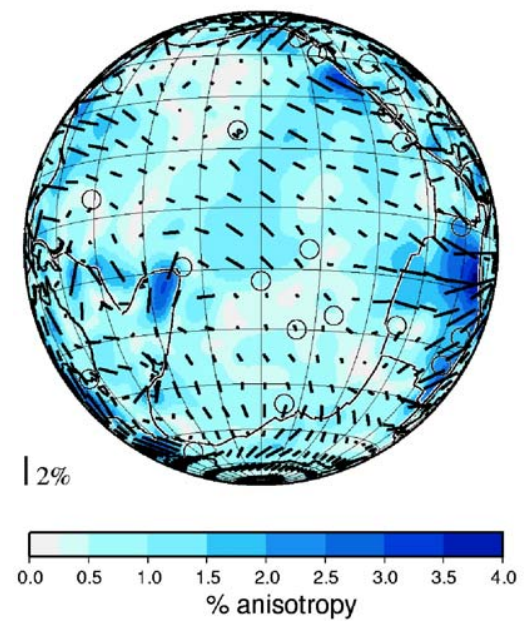

anisotropy from multi-mode surface waveform tomography may act as a further, depth-dependent constraint on subduction zone flow models.

\subsection{Comparison with radial anisotropy}

Up to now we have only discussed the SV wave azimuthal anisotropy inferred from Rayleigh waves. However, the lattice preferred orientation of anisotropic crystals in the upper mantle is also expected to produce radial or polarisation anisotropy, which results in the Love-Rayleigh wave discrepancy (Love wave phase dispersion curves are inconsistent with those predicted by isotropic Earth models that account for Rayleigh wave phase dispersion). Radial anisotropy with $v_{\mathrm{SH}}>v_{\mathrm{Sv}}$ has been observed globally up to $220 \mathrm{~km}$ depth, and is included in the 1D PREM model [38]. Generation of radial anisotropy of this sign by LPO requires that the fast axes of intrinsically anisotropic crystals lie preferentially in the horizontal plane, but it does not require any particular alignment within the plane.

The amplitude of both radial and azimuthal anisotropy depends in general on the geometry and fabric strength of the LPO [54]. Regions in which LPO is caused by simple shear associated with plate motion are expected to develop classical orthorhombic fabric, with the ' $a$ ' axes close to the flow direction and the ' $b$ ' axes concentrated normal to the flow plane, leading to strong azimuthal and radial $\left(v_{\mathrm{SH}}>v_{\mathrm{SV}}\right)$ seismic anisotropy. In the case of olivine LPO with ' $a$ ' axes randomly oriented in the horizontal plane (the case of a transversely isotropic medium with vertical axis of symmetry), the resulting $v_{\mathrm{sv}}$ azimuthal anisotropy in the horizontal

(b) Radial anisotropy

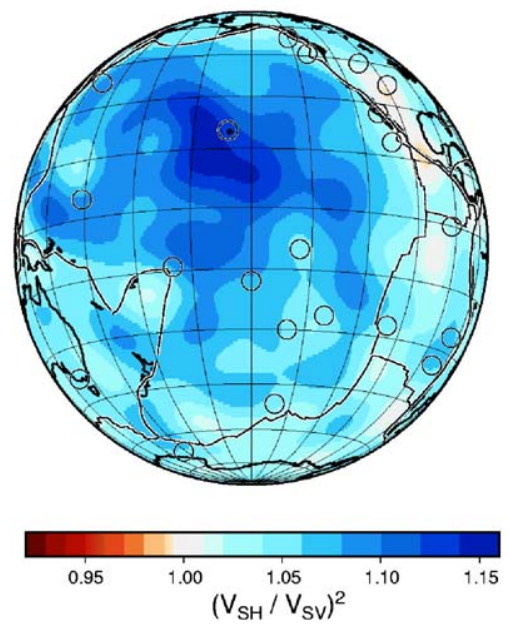

Fig. 11. Comparison between (a) our azimuthal anisotropy results and (b) the radial anisotropy results of [65], re-plotted to show $\xi=\left(v_{\mathrm{SH}} / v_{\mathrm{sv}}\right)^{2}$. 
plane is notably reduced, but the medium presents radial anisotropy with $v_{\mathrm{SH}}>v_{\mathrm{sv}}$, even if the ' $b$ ' axes are strongly concentrated in the vertical direction [64]. In this case, if the distances over which the fast axes vary in orientation are smaller than the lateral resolution of the surface waves, azimuthal anisotropy is likely to be averaged out, resulting in a weak or null anisotropic signal.

Although it is not possible to extract radial anisotropy information from a data-set such as ours, composed entirely of Rayleigh waves, we can check the consistency of our azimuthal anisotropy results with published maps of radial anisotropy in the Pacific region. Ekström and Dziewonski [65] have published a high resolution radially anisotropic model of the Pacific upper mantle, in which they observe a broad anomaly in radial anisotropy in the northern Pacific, with its centre near Hawaii. The amplitude of this anomaly, which reaches a maximum of $\xi=$ $\left(v_{\mathrm{SH}}^{2} / v_{\mathrm{Sv}}^{2}\right)$ of 1.15 at $150 \mathrm{~km}$ depth (Fig. 11b), is significantly larger than $\xi$ of 1.06 obtained for Pacific plate mantle of the same age by Nishimura and Forsyth [8]. Such a strong anisotropy is somewhat difficult to interpret: if we assume an average pyrolitic mantle composition, and the anisotropic parameters tabulated by Estey and Douglas [50], a $\xi$ value of 1.14 implies that $100 \%$ of the anisotropic crystals are oriented in the horizontal plane (if we assume an olivine only mantle composition, the $\xi$ value corresponding to $100 \%$ horizontal alignment is 1.3). It is hard to imagine a physical process that would induce such an extreme degree of alignment. The Ekström and Dziewonski [65] observation has recently been confirmed [66], indicating that the firstorder physical interpretation of the amplitude of radial anisotropy in terms of the percentage of horizontally aligned crystals may no longer be adequate, or that we may require better estimates of mantle composition and/or of anisotropic elastic parameters of mantle minerals.

In Fig. 11, we compare the relative amplitudes and geographic distribution of our azimuthal anisotropy results and the radial anisotropy observed by Ekström and Dziewonski [65] at $150 \mathrm{~km}$ depth. The broad region with strong radial anisotropy observed by [65] covers areas with both strong (region between Hawaii and the Samoa, Cook and Tahiti hot-spots) and weak azimuthal anisotropy (west and northwest of Hawaii). Our azimuthal coverage (Fig. 1) and synthetic tests (Fig. 5) indicate that the juxtaposition of strong plate motion related azimuthal anisotropy SE of Hawaii to weak azimuthal anisotropy NW of Hawaii is likely to be a real feature. This distribution of azimuthal anisotropy results can be qualitatively reconciled with the $v_{\mathrm{SH}}>v_{\mathrm{Sv}}$ radial anisotropy of [65] by invoking plate motion driven shear flow for the region lying SE of Hawaii, and by invoking perturbations due to a plume related mantle upwelling for the region lying NW of Hawaii, downstream with respect to the APM-induced flow direction. In a number of other regions, our model displays strong, coherent azimuthal anisotropy where [65] find only weak $v_{\mathrm{SH}}>v_{\mathrm{Sv}}$ radial anisotropy. Examples include the eastern Nazca plate, the Pacific plate boundaries near the coasts of California, Alaska and Japan, and the region between the new Hebrides and Fiji, all regions closely associated with recent or present subduction. Subduction zone processes may be responsible for generating strong and azimuthally coherent LPO out of the horizontal plane, which may lead to the observed combination of significant azimuthal and weak radial anisotropy. This comparison suggests that the geographic distributions of azimuthal and radial anisotropy are qualitatively compatible, although their relative amplitudes are not always straightforwardly reconciled.

\section{Conclusion}

We have presented in detail the azimuthally anisotropic results of a high resolution Rayleigh wave tomography study, the isotropic component of which is described in [1]. Our results are fully consistent with the hypothesis of anisotropy generation in the Earth's mantle by preferential alignment of anisotropic crystals in response to the shear strains induced by mantle flow.

At lithospheric depths, we have found a good agreement between fast azimuthal anisotropy orientations and paleo ridge spreading directions as recorded by seafloor magnetic anomalies. At asthenospheric depths we have found a strong correlation between fast azimuthal anisotropy orientations and the directions of current plate motions, consistent with lattice preferred orientation due to the simple shear imposed on the asthenosphere by tectonic plates. We have observed perturbations to the asthenospheric pattern of seismic anisotropy in proximity to Pacific hot-spots; these perturbations agree qualitatively with the predictions of numerical models of LPO generation in plume-disturbed plate motion-driven flow [60], suggesting that perturbations in the pattern of oceanic azimuthal anisotropy may provide indirect evidence of the presence of thin mantle upwellings.

\section{Acknowledgements}

A. Maggi was supported by a Marie Curie Individual Fellowship (contract HPMF-CT-2002-01636) from the European Union. The study was also supported by the DyETI program 'Imagerie globale et implications pour la dynamique de la zone de transition' funded by the French Institut National des Sciences de l'Univers 
(INSU). The facilities of the IRIS Data Management System, and specifically the IRIS Data Management Center, were used for access to waveform and metadata required in this study. The IRIS DMS is funded through the National Science Foundation and specifically the GEO Directorate through the Instrumentation and Facilities Program of the National Science Foundation under Cooperative Agreement EAR-0004370. Additional waveform data were obtained from GEOSCOPE and from the PLUME broadband deployment in the South Pacific, funded by the French Ministère de la Recherche, and facilitated by the Centre National de la Recherche Scientifique (CNRS), by the government of French Polynesia, and by the Université de Polynesie française (UPF). Supercomputer facilities were provided by the IDRIS national center in France. Most figures in this paper were prepared using the open source GMT software developed and maintained by Paul Wessel andWalter Smith, and supported by the NSF. The authors would like to thank Jean-Jacques Lévêque for helpful discussions on anisotropy, Fabrice Fontaine and D. Reymond for supplying the PLUME data, and Jérôme Dyment for providing a digitised version of the magnetic anomalies from [53].

\section{References}

[1] A. Maggi, E. Debayle, K. Priestley, G. Barruol, Multi-mode surface waveform tomography of the Pacific Ocean: a close look at the lithospheric cooling signature, Geophys. J. Int. 166 (3) (2006), doi:10.1111/j.1365-246x.2006.03037.x.

[2] H. Hess, Seismic anisotropy of the uppermost mantle under oceans, Nature 203 (1964) 629-631.

[3] R. Raitt, G. Shor, T. Francis, G. Morris, Anisotropy of the Pacific upper mantle, J. Geophys. Res. 74 (1969) 3095-3109.

[4] L. Vinnik, G. Kosarev, L. Makeyeva, Anisotropy of the lithosphere from the observation of $S K S$ and SKKS, Proc. Acad. Sci. USSR 278 (1984) 1335-1339 in Russian.

[5] K. Aki, K. Kaminuma, Phase velocity in Japan. Part I. Love waves from the Aleutian shock of March 1957, Bull. Earthq. Res. Inst. 41 (1963) 243-259.

[6] T. McEvilly, Central U.S. crust-upper mantle structure from Love and Rayleigh wave phase velocity inversion, Bull. Seismol. Soc. Am. 54 (1964) 1997-2015.

[7] J.-P. Montagner, N. Jobert, Vectorial tomography - II: application to the Indian Ocean, Geophys. J. 94 (1988) 309-344.

[8] C. Nishimura, D. Forsyth, The anisotropic structure of the upper mantle in the Pacific, Geophys. J. 96 (1989) 203-229.

[9] A. Nicolas, N. Christensen, Formation of anisotropy in upper mantle peridotites: a review, in: K. Fuchs, C. Froidevaux (Eds.), Composition, Structure and Dynamics of the Lithosphere-Asthenosphere System, Vol. 16 of Geodynamics, American Geophysical Union, Washington, D.C., 1987, pp. 111-123.

[10] D. Mainprice, G. Barruol, W. Ben Ismail, The seismic anisotropy of the Earth's mantle: from single crystal to polycrystal, in: S.-I. Karato, A. Forte, R. Libermann, G. Masters, L. Siztrude (Eds.), Earth's Deep Interior: Mineral Physics and Tomography from the Atomic to the
Global Scale, Geophysical Monograph, vol. 117, American Geophysical Union, Washington, D.C., 2000, pp. 237-264.

[11] S. Zhang, S. Karato, Lattice preferred orientation of olivine aggregates deformed in simple shear, Nature 375 (6534) (1995) 774-777.

[12] A. Tommasi, Forward modeling of the development of seismic anisotropy in the upper mantle, Earth Planet. Sci. Lett. 160 (1998) 1-13.

[13] H. Jung, S.-I. Karato, Water-induced fabric transitions in olivine, Science 293 (2001) 1460-1463.

[14] E. Kaminski, The influence of water on the development of lattice preferred orientation in olivine aggregates, Geophy. Res. Lett. 29 (12), doi:10.1029/2002GL014710.

[15] C. Beghein, J. Trampert, Probability density functions for radial anisotropy: implications for the upper $1200 \mathrm{~km}$ of the mantle, Earth Planet. Sci. Lett. 217 (1-2) (2004) 151-162.

[16] D. McKenzie, Finite deformation during fluid flow, Geophys. J. R. Astron. Soc. 58 (1979) 689-715.

[17] N. Ribe, Seismic anisotropy and mantle flow, J. Geophys. Res. 94 (1989) 4213-4223.

[18] C. Hall, K. Fischer, E. Parmentier, D. Blackman, The influence of plate motions on three-dimensional back arc mantle flow an shear wave splitting, J. Geophys. Res. 105 (B12) (2000) 28,009-28,033.

[19] D. Blackman, J.-M. Kendall, Seismic anisotropy of the upper mantle: 2. Predictions for current plate boundary flow models, Geochem. Geophys. Geosyst. 3, $2001 \mathrm{GC} 000247$.

[20] T. Becker, J. Kellogg, G. Ekström, R. O'Connell, Comparison of azimuthal seismic anisotropy from surface waves and finite strain from global mantle circulation models, Geophys. J. Int. 155 (2003) 696-714.

[21] C. Gaboret, A. Forte, J.-P. Montagner, The unique dynamics of the Pacific Hemisphere mantle and its signature on seismic anisotropy, Earth Planet. Sci. Lett. 208 (2003) 219-233.

[22] D. Forsyth, The early structural evolution and anisotropy of the oceanic upper mantle, Geophys. J. R. Astron. Soc. 43 (1975) $103-162$.

[23] C. Nishimura, D. Forsyth, Rayleigh wave phase velocities in the Pacific with implications for azimuthal anisotropy and lateral heterogeneities, Geophys. J. 94 (1988) 479-501.

[24] J.-P. Montagner, T. Tanimoto, Global upper mantle tomography of seismic velocites and anisotropies, J. Geophys. Res. 96 (1991) 20,337-20,351.

[25] J.-P. Montagner, Upper mantle low anisotropy channels below the Pacific Plate, Earth Planet. Sci. Lett. 202 (2002) 263-274.

[26] J. Trampert, J. Woodhouse, Global anisotropic phase velocity maps for fundamental mode surface waves between 40 and $150 \mathrm{~s}$, Geophys. J. Int. 154 (2003) 154-165.

[27] D. Smith, M. Ritzwoller, N. Shapiro, Stratification of anisotropy in the Pacific upper mantle, J. Geophys. Res. 109, doi:10.1029/ 2004JB003200.

[28] E. Beucler, J.-P. Montagner, Computation of large anisotropic seismic heterogeneities (CLASH), Geophys. J. Int. 165 (2006) 447-468.

[29] M. Cara, J. Lévêque, Waveform inversion using secondary observables, Geophys. Res. Lett. 14 (1987) 1046-1049.

[30] E. Debayle, SV-wave azimuthal anisotropy in the Australian upper mantle: preliminary results from automated Rayleigh waveform inversion, Geophys. J. Int. 137 (1999) 747-754.

[31] J. Lévêque, E. Debayle, V. Maupin, Anisotropy in the Indian Ocean upper mantle from Rayleigh and Love waveform inversion, Geophys. J. Int. 133 (1998) 529-540.

[32] E. Debayle, M. Sambridge, Inversion of massive surface wave data sets: model construction and resolution assessment, J. Geophys. Res. 109, doi:10.1029/2003JB002652. 
[33] G. Barruol, et al., PLUME investigates the South Pacific Superswell, EOS Trans. AGU 83 (2002) 511-514.

[34] E. Debayle, B. Kennett, The Australian continental upper mantle: structure and deformation inferred from surface waves, J. Geophys. Res. 105 (2000) 25423-25450.

[35] S. Pilidou, K. Priestley, O. Gudmundsson, E. Debayle, Upper mantle $s$-wave speed heterogeneity and anisotropy beneath the north Atlantic from regional surface wave tomography: the Iceland and Azores plumes, Geophys. J. Int. 159 (3) (2004) 1057-1076.

[36] E. Debayle, M. Sambridge, K. Priestley, Global azimuthal seismic anisotropy and the unique plate-motion deformation of Australia, Nature 433 (2005) 509-512.

[37] K. Priestley, D. McKenzie, E. Debayle, The state of the upper mantle beneath southern Africa, Tectonophysics 416 (2006) 101-112.

[38] M. Dziewonski, A.D. Anderson, Preliminary Reference Earth Model, Phys. Earth Planet. Inter. 25 (4) (1981) 297-356.

[39] H. Nataf, Y. Ricard, 3SMAC: an a priori tomographic model of the upper mantle based on geophysical modeling, Phys. Earth Planet. Inter. 95 (1995) 101-122.

[40] J.-P. Montagner, Regional three-dimensional structures using long-period surface waves, Ann. Geophys. 4 (1986) 283-294.

[41] M. Smith, F. Dahlen, The azimuthal dependence of Love and Rayleigh wave propagation in a slightly anisotropic medium, J. Geophys. Res. 78 (1973) 3321-3333.

[42] J.-P. Montagner, H. Nataf, A simple method for inverting the azimuthal anisotropy of surface waves, J. Geophys. Res. 91 (1986) $511-520$

[43] B. Kennett, K. Yoshizawa, A reappraisal of regional surface wave tomography, Geophys. J. Int. 150 (1) (2002) 37-44.

[44] J. Spetzler, R. Snieder, The effect of small-scale heterogeneity on the arrival time of waves, Geophys. J. Int. 145 (2001) 786-796.

[45] K. Yoshizawa, B. Kennett, Determination of the influence zone for surface wave paths, Geophys. J. Int. 149 (2002) 440-453.

[46] A. Sieminski, E. Debayle, J.-J. Lévêque, Seismic evidence for deep low-velocity anomalies in the transition zone beneath Western Antarctica, Earth Planet. Sci. Lett. 216 (2003) 645-661 doi:10.1016/S0012-821X(03)00518-1.

[47] N. Christensen, The magnitude, symmetry and origin of upper mantle anisotropy based on fabric analyses of ultramafic tectonites, Geophys. J. R. Astron. Soc. 76 (1) (1984) 89-111.

[48] C. Wolfe, S. Solomon, Shear-wave splitting and implications for mantle flow beneath the MELT region of the East Pacific Rise, Science 280 (1998) 1230-1232.

[49] N. Harmon, D. Forsyth, K. Fischer, S. Sebb, Variations in shearwave splitting in young Pacific seafloor, Geophy. Res. Lett. 31 (L15609), doi:10.1029/2004GL020495.

[50] L. Estey, B. Douglas, Upper mantle anisotropy: a preliminary model, J. Geophys. Res. 91 (B11) (1986) 11,393-11,406.

[51] N. Ribe, On the relation between seismic anisotropy and finite strain, J. Geophys. Res. 97 (1992) 8737-8747.

[52] A. Tommasi, D. Mainprice, G. Canova, Y. Chastel, Viscoplastic self-consistent and equilibrium-based modeling of olivine lattice preferred orientations: implications for the upper mantle seismic anisotropy, J. Geophys. Res. 104 (B4) (2000) 7893-7908.

[53] S. Cande, J. LaBrecque, R. Larson, W. Pitmann, X. Golovchenko, W. Haxby, Magnetic lineations of the world's ocean basins, map Series (1989).

[54] W. Ben Ismail, D. Mainprice, An olivine fabric database; an overview of upper mantle fabrics and seismic anisotropy, Tectonophysics 296 (1998) 145-157.

[55] B. Ildefonse, F. Boudier, A. Nicolas, Asthenospheric and lithospheric deformation in Oman ophiolitic peridotite, in: A. Snoke, J. Tullis, V. Todd (Eds.), Fault related rocks. A petrographic atlas, Princeton University Press, 1998, pp. 588-591.

[56] A. Nicolas, F. Boudier, B. Ildefonse, E. Ball, Accretion of Oman ophiolite and United Emirates ophiolite. Discussion of a new structural map, Mar. Geophys. Res. 21 (2000) 147-179.

[57] J. Mammerickx, G. Sharman, Tectonic evolution of the North Pacific during the Cretaceous quiet period, J. Geophys. Res. 93 (1988) 3009-3024.

[58] C. Mayes, L. Lawver, D. Sandwell, Tectonic history and new isochron chart of the South Pacific, J. Geophys. Res. 95 (1990) $8543-8567$.

[59] C. Thoraval, A. Tommasi, M. Doin, Plume-lithosphere interaction beneath a fast moving plate, Geophy. Res. Lett. 33, doi:10.1029/2005GL024047.

[60] E. Kaminski, N. Ribe, Timescales for the evolution of seismic anisotropy in mantle flow, Geochem. Geophys. Geosyst. 3 (8), doi:10.1029/2001GC000222.

[61] G. Barruol, R. Hoffmann, Upper mantle anistropy beneath Geoscope stations, J. Geophys. Res. 104 (B5) (1999) $10,757-10,773$.

[62] K. Fischer, D. Wiens, The depth distribution of mantle anisotropy beneath the Tonga subduction zone, Earth Planet. Sci. Lett. 142 (1996) 253-260.

[63] T. Lassak, M. Fouch, C. Hall, E. Kaminski, Seismic characterization of mantle flow in subduction zone systems: can we resolve a hydrated mantle wedge? Earth Planet. Sci. Lett. 243 (2006) 632-649.

[64] A. Tommasi, B. Tikoff, A. Vauchez, Upper mantle tectonics: three-dimensional deformation, olivine crystallographic fabrics and seismic properties, Earth Planet. Sci. Lett. 168 (1999) 173-186.

[65] G. Ekström, A. Dziewonski, The unique anisotropy of the Pacific upper mantle, Nature 394 (1998) 168-172.

[66] L. Boschi, G. Ekström, New images of the Earth's upper mantle from measurements of surface wave phase velocity anomalies, J. Geophys. Res. 107 (B4), doi:10.1029/2000JB000059.

[67] V. Courtillot, A. Davaille, J. Besse, J. Stock, Three distinct types of hotspot in the Earth's mantle, Earth Planet. Sci. Lett. 205 (2003) 295-308.

[68] J. Ritsema, R. Allen, The elusive mantle plume, Earth Planet. Sci. Lett. 207 (2003) 1-12. 